OPEN ACCESS

Edited by:

Pamela Imperadore,

Department of Biology and Evolution of Marine Organisms, Zoological

Station Anton Dohrn, Italy

Reviewed by:

Matthew B. Veldman,

Medical College of Wisconsin

United States

Jerry Silver,

Case Western Reserve University,

United States

Karen Echeverri,

Marine Biological Laboratory (MBL),

United States

${ }^{*}$ Correspondence:

Jianli Hu

jianli.hu@temple.edu

Michael E. Selzer

michael.selzer@temple.edu

Specialty section:

This article was submitted to Morphogenesis and Patterning,

a section of the journal

Frontiers in Cell and Developmental

Biology

Received: 14 January 2021

Accepted: 26 February 2021

Published: 25 March 2021

Citation:

Hu J, Rodemer W, Zhang G, Jin L-Q, Li S and Selzer ME (2021) Chondroitinase $A B C$ Promotes Axon

Regeneration and Reduces

Retrograde Apoptosis Signaling

in Lamprey.

Front. Cell Dev. Biol. 9:653638. doi: 10.3389/fcell.2021.653638

\section{Chondroitinase ABC Promotes Axon Regeneration and Reduces Retrograde Apoptosis Signaling in Lamprey}

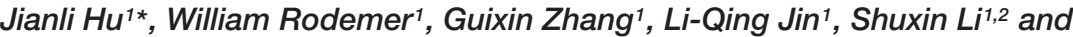 \\ Michael E. Selzer ${ }^{1,3 *}$
}

\begin{abstract}
'Shriners Hospitals Pediatric Research Center (Center for Neural Repair and Rehabilitation), Lewis Katz School of Medicine at Temple University, Philadelphia, PA, United States, ${ }^{2}$ Department of Anatomy and Cell Biology, Lewis Katz School of Medicine at Temple University, Philadelphia, PA, United States, ${ }^{3}$ Department of Neurology, Lewis Katz School of Medicine at Temple University, Philadelphia, PA, United States
\end{abstract}

Paralysis following spinal cord injury (SCI) is due to failure of axonal regeneration. It is believed that axon growth is inhibited by the presence of several types of inhibitory molecules in central nervous system (CNS), including the chondroitin sulfate proteoglycans (CSPGs). Many studies have shown that digestion of CSPGs with chondroitinase $\mathrm{ABC}(\mathrm{Ch} \mathrm{ABC})$ can enhance axon growth and functional recovery after $\mathrm{SCl}$. However, due to the complexity of the mammalian CNS, it is still unclear whether this involves true regeneration or only collateral sprouting by uninjured axons, whether it affects the expression of CSPG receptors such as protein tyrosine phosphatase sigma (PTP $\sigma)$, and whether it influences retrograde neuronal apoptosis after $\mathrm{SCl}$. In the present study, we assessed the roles of CSPGs in the regeneration of spinal-projecting axons from brainstem neurons, and in the process of retrograde neuronal apoptosis. Using the fluorochrome-labeled inhibitor of caspase activity (FLICA) method, apoptotic signaling was seen primarily in those large, individually identified reticulospinal (RS) neurons that are known to be "bad-regenerators." Compared to uninjured controls, the number of all RS neurons showing polycaspase activity increased significantly at 2, 4, 8, and 11 weeks post-transection (post-TX). ChABC application to a fresh TX site reduced the number of polycaspase-positive RS neurons at 2 and 11 weeks post-TX, and also reduced the number of active caspase 3-positive RS neurons at 4 weeks postTX, which confirmed the beneficial role of ChABC treatment in retrograde apoptotic signaling. ChABC treatment also greatly promoted axonal regeneration at 10 weeks post-TX. Correspondingly, PTPo mRNA expression was reduced in the perikaryon. Previously, PTPo mRNA expression was shown to correlate with neuronal apoptotic signaling at 2 and 10 weeks post-TX. In the present study, this correlation persisted after ChABC treatment, which suggests that PTP $\sigma$ may be involved more generally in signaling axotomy-induced retrograde neuronal apoptosis. Moreover, ChABC treatment 
caused Akt activation (pAkt-308) to be greatly enhanced in brain post-TX, which was further confirmed in individually identified RS neurons. Thus, CSPG digestion not only enhances axon regeneration after SCl, but also inhibits retrograde RS neuronal apoptosis signaling, possibly by reducing PTPo expression and enhancing Akt activation.

Keywords: ChABC, neuronal death, axon regeneration, PTP, , Akt, lamprey, SCI, FLICA

\section{INTRODUCTION}

Paralysis following spinal cord injury (SCI) is due to axon interruption and failure of regeneration. Accumulating evidence suggests that both extrinsic and intrinsic factors contribute to the inability of axons to regenerate. Among the key factors, chondroitin sulfate proteoglycans (CSPGs) are normal constituents of the perineuronal nets in central nervous system (CNS; Bruckner et al., 2000; Deepa et al., 2006), and are greatly elevated after SCI, both in rodents (Bradbury et al., 2002) and lampreys (Zhang et al., 2014). In the lamprey, CSPG levels peaked near the transection (TX) at 2 weeks post-TX, returning to normal by 10 weeks. In vitro experiments showed that CSPGs can inhibit neurite outgrowth (Snow et al., 1990; McKeon et al., 1991), while digestion of CSPGs with chondroitinase $\mathrm{ABC}$ (ChABC) can prevent macrophage-induced axon retraction (Busch et al., 2009). $\mathrm{ChABC}$ application in vivo leads to axon sprouting in the intact spinal cord (Galtrey et al., 2007). Moreover, intrathecal ChABC promoted growth of spinal axons and functional recovery in rats (Bradbury et al., 2002). Transgenic ChABC-mediated digestion of the CSPGs promoted growth of axons past a dorsal root crush (Cafferty et al., 2007), enhanced sensory recovery (Cafferty et al., 2008) and promoted compensatory sprouting and functional recovery after unilateral corticospinal tract (CST) lesion (Starkey et al., 2012). The molecular mechanisms for these effects are not certain, and the behavior of growth cones in vitro may not represent mechanisms of regeneration of mature axons in vivo (Jin et al., 2009). Although CSPGs might interfere with axon adhesion to extracellular matrix (Friedlander et al., 1994), the receptor-like protein tyrosine phosphatases (RPTPs), protein tyrosine phosphatase sigma (PTP $\sigma)$ and leukocyte common antigen-related phosphatase (LAR), have been identified as transmembrane receptors for CSPGs (Shen et al., 2009; Fisher et al., 2011; Sharma et al., 2012). Genetic disruption of PTP $\sigma$ promoted axon growth into CSPG-rich regions of SCI (Shen et al., 2009), and transgenic deletion of LAR increased growth of descending axons caudal to the lesion and enhanced locomotor recovery after SCI (Xu et al., 2015). This also was true for systemic injection of small peptide inhibitors of LAR (Fisher et al., 2011) and PTPб (Lang et al., 2015). RPTPs exist in the plasma membrane as auto-inhibited dimers. When bound to ligands, they separate, triggering phosphatase activity (Hower et al., 2009). Similar to myelin-associated growth inhibitors such as Nogo (Monnier et al., 2003), CSPG-mediated inhibition of neurite growth appears to involve RhoA activation (Fisher et al., 2011).

Although removal of the polysaccharide side chains of CSPGs with $\mathrm{ChABC}$ enhances axon growth and functional recovery after SCI in mammalian partial injury models, it is not clear whether this involves true regeneration of injured axons, or to collateral sprouting by spared axons. Nor is it known how this treatment affects the expression of CSPG receptors and their downstream signaling pathways. Since lampreys have both CSPGs and their RPTPs, to get around the limitations of mammalian models, we used complete TX of lamprey spinal cord to determine whether these effects relate to true regeneration of lesioned axons.

In the lamprey, the main descending system that transmits commands from the brain to the spinal cord is composed of reticulospinal (RS) neurons, which are responsible for initiation of locomotion, steering, and equilibrium control (Deliagina et al., 2000). The 18 pairs of individually identified RS neurons have axons that extend the entire length of spinal cord and therefore, are always axotomized by a complete spinal cord TX. The perikarya of these identified RS neurons can be labeled retrogradely by application of a dye to the site of the fresh TX. In the lamprey, some spinal-projecting neurons are good regenerators and some are bad (Davis and McClellan, 1994; Jacobs et al., 1997). The latter often experience a very delayed form of apoptosis (Shifman et al., 2008; Busch and Morgan, 2012; Hu et al., 2013, 2017). In the present study, these features have been used to determine the effects of ChABC treatment on the regeneration of axons belonging to spinal-projecting neurons, on their retrograde death after spinal cord TX, and on a downstream pathway thought to participate in these effects. In vitro studies had indicated that Akt is an important downstream signaling molecule of CSPG receptors (Fisher et al., 2011). In addition, the deletion of phosphatase and tensin homolog (PTEN) has been reported to promote potent CNS axon regeneration after optic nerve injury (Park et al., 2008) and SCI (Liu et al., 2010; Du et al., 2015), and the effect of PTEN knockdown to promote survival of retinal ganglion cells (RGC) and regeneration of their axons appeared to involve activation of Akt (Yang et al., 2014; Guo et al., 2016). Therefore, we explored the role of Akt in mediating the axon regeneration and suppression of retrograde neuronal death produced by ChABC treatment after SCI in the lamprey.

\section{MATERIALS AND METHODS}

\section{Spinal Cord Transection, ChABC Treatment, and Retrograde Labeling}

Wild-type larval lampreys, Petromyzon marinus, 10-14 cm in length (4-5 years old), were obtained from streams of Lake Michigan and maintained in fresh water tanks at room temperature (RT) until use. All animal procedures described in this report were performed with approval from the Temple 
University Institutional Animal Care and Use Committee (ACUP\#: 4922). For spinal cord TX, animals were anesthetized by immersion in $0.1 \%$ tricaine methanesulfonate, and the spinal cord was exposed by an incision along the dorsal midline at the level of the fifth gill. TX of the spinal cord was performed with Castroviejo scissors. Completeness of TX was confirmed by retraction and visual inspection of the cut ends. For ChABC treatment, $1 \mu \mathrm{l} \mathrm{ChABC} \mathrm{(Cat \#} \mathrm{C2905,} \mathrm{Sigma-Aldrich)} \mathrm{dissolved} \mathrm{in}$ enzyme buffer was applied to the TX site and a pledget of Gelfoam soaked with $1 \mu \mathrm{l}$ ChABC was placed gently on the surface of the spinal cord spanning the injury site. Control animals were treated with enzyme buffer. To label axon tips after SCI, we placed a pledget of Gelfoam soaked in 5\% dextran tetramethylrhodamine (DTMR; Cat\# D1817, Thermo Fisher Scientific) with enzyme buffer or ChABC into the TX gap. TXed lampreys recovered on ice for $2 \mathrm{~h}$ and then were returned to fresh water tanks at RT for 1 , $2,4,8$, and 11 weeks, at which times the brainstems were removed for fluorochrome-labeled inhibitor of caspase activity (FLICA) assay and then followed with PTP $\sigma$ mRNA in situ hybridization (ISH). The spinal cords were carefully dissected out at 2 weeks and fixed with $4 \%$ paraformaldehyde (PFA) immediately. After washing with phosphate buffered saline (PBS), the axon tips were imaged with a fluorescence microscope. To assess axon regeneration at 10 weeks after the first TX at 5th gill, we performed the second TX at $5 \mathrm{~mm}$ caudal to the first TX and placed a pledget of Gelfoam soaked in 5\% DTMR into the 2nd TX gap. The lampreys recovered on ice for $2 \mathrm{~h}$ and then were returned to fresh water tanks at RT for another 1 week to allow the DTMR to label the regenerated axons (Figures 2E-I). To retrogradely label the individual RS neurons for the convenience of pAkt-308 immunostaining analysis, 5\% dextran Alexa Fluor 488 (DAF-488, Cat\# D22910, Thermo Fisher Scientific) was placed into the TX gap. TXed lampreys recovered on ice for $2 \mathrm{~h}$ and then were returned to fresh water tanks at RT for 2, 4 , or 8 weeks, at which times the brainstems were removed for pAkt-308 immunofluorescence staining.

\section{FLICA on Whole-Mounted Lamprey Brains}

After the recovery times described above, animals were reanesthetized by immersion in saturated benzocaine solution. Brains were dissected out in ice-cold lamprey Ringer's buffer (110 mM NaCl, $2.1 \mathrm{mM} \mathrm{KCl,} 2.6 \mathrm{mM} \mathrm{CaCl}_{2}, 1.8 \mathrm{mM} \mathrm{MgCl}_{2}$, and $10 \mathrm{mM}$ Tris buffer; $\mathrm{pH}$ 7.4). The posterior and cerebrotectal commissures of the freshly dissected brains were split along the dorsal midline. Brains were incubated immediately at $4^{\circ} \mathrm{C}$ for $1 \mathrm{~h}$ in $150 \mu \mathrm{L} 1 \times$ FLICA labeling solution (Image-iT $\mathrm{T}^{\mathrm{TM}}$ Live Green Poly-Caspases Detection Kit, Cat\# I35104, Molecular Probes; or Green Caspase-3 Staining Kit, Cat\# PK-CA577-K18325, PromoKine), which was diluted with PBS. Afterward, brains were washed five times with $1 \mathrm{X}$ wash buffer on a rotator at $4^{\circ} \mathrm{C}$, 5 min per time. The alar plates of brains were deflected laterally and pinned flat to a small strip of Sylgard (Dow Corning Co., United States). The tissue was fixed in 4\% PFA in PBS for $2 \mathrm{~h}$ at RT, and then washed three times in PBS at RT. Fluorescence images of brains were captured immediately with a Nikon $80 \mathrm{i}$ microscope. The whole procedure was conducted in the dark and all the samples were carefully protected from light. The brains were placed in $70 \% \mathrm{EtOH}$ and kept at $-20^{\circ} \mathrm{C}$ for PTP $\sigma$ mRNA ISH. Control experiments were performed using brains from lampreys without spinal cord TX. All images were acquired using the same parameters.

\section{In situ Hybridization on Whole-Mounted Lamprey Brains After FLICA}

In situ hybridization was carried out according to modifications of the chromogenic method previously described (Swain et al., 1994). The whole-mounted brains were placed in Eppendorf tubes and washed in PTW (0.1\% Tween-20 in PBS), then prehybridized at $\sim 55^{\circ} \mathrm{C}$ in hybridization solution for $60 \mathrm{~min}$ (50\% deionized formamide, 5X SSC, $100 \mathrm{mg} / \mathrm{ml}$ Torula yeast RNA, $100 \mathrm{mg} / \mathrm{ml}$ wheat germ tRNA, $50 \mathrm{mg} / \mathrm{ml}$ heparin, $0.1 \%$ Tween-20). Hybridization was carried out by adding digoxinlabeled PTP $\sigma$ antisense RNA probes, $1 \mu \mathrm{g} / \mathrm{ml}$ in hybridization solution, to the brain samples, which were kept at $\sim 55^{\circ} \mathrm{C}$ on a rotator overnight. Brains were washed in hybridization solution at $55^{\circ} \mathrm{C}$ followed by washes in PTW and PBT ( $0.1 \%$ bovine serum albumin, $0.2 \%$ Triton X-100 in PBS) at RT. Anti-DigoxigeninAP Fab fragments (Cat\# 11093274910, Roche Applied Science) were applied $1: 1,000$ to the brain samples at $4^{\circ} \mathrm{C}$ on a rotator overnight. The samples were washed sequentially in PBT and SMT (100 mM NaCl, $50 \mathrm{mM} \mathrm{MgCl2,} 100 \mathrm{mM}$ Tris, $\mathrm{pH}$ 9.5, $0.1 \%$ Tween-20). The chromogenic reaction was carried out in a solution containing $20 \mu \mathrm{l}$ of NBT/BCIP stock solution (Cat\# 11681451001, Roche Applied Science) in each $1 \mathrm{ml}$ of SMT on ice in the dark for $1 \mathrm{~h}$, or until the reaction was completed, as determined by monitoring under a dissecting microscope. Finally, brain samples were washed in PBS, and the meninges were stripped gently from the posterior surface of the brain, using a forceps under a dissecting microscope. The brains were mounted onto glass slides and bright-field images were captured with a Nikon $80 \mathrm{i}$ microscope.

\section{Calculation of Probabilities of FLICA- and PTP $\sigma$-Positive Identified RS Neurons}

The RS neurons were identified individually in brain wholemounts labeled retrogradely with DTMR, based on their characteristic morphologies, sizes, and locations (Jacobs et al., 1997). Thus far, there are no molecular markers specific for individual RS neurons, although retrograde labeling combined with immunohistochemistry for developmentally regulated genes has been used to study the segmental development of the embryonic lamprey hindbrain (Murakami et al., 2004). The number of $\mathrm{PTP} \sigma$-positive neurons were counted separately for each of the individually identified RS neurons in each brain. Then, for each of the individually identified RS neurons, the number of РТР $\sigma$-positive neurons was divided by the total number of neurons (2) of that individual type (РTP $\sigma$-positive and -negative) in each brain, and the percentages were considered the probability of PTP $\sigma$ positivity for each of the identified RS neurons. For example, among the five brains treated with $\mathrm{ChABC}$ and surveyed at 2 weeks post-TX, three M1 neurons were PTP $\sigma$ positive. Since each brain has two M1 neurons, the total number of M1 neurons was $2 \times 5=10$. Thus, the probability of PTP $\sigma$ 
positivity for the M1 neuron in this ChABC-treated group was $(3 \div 10) \times 100=30 \%$. This method also was used to calculate the percent of FLICA-positive identified RS neurons.

\section{Western Blotting}

The brains were collected from lampreys under a dissecting microscope. To investigate the expression of Akt in brains (with or without ChABC treatment), we dissected out the brain from the olfactory lobe to the obex. The tissues were snap-frozen in liquid nitrogen and homogenized in cold lysis buffer (Cat\# C3228, Sigma-Aldrich) supplemented with $1 \times$ protease inhibitor cocktail (Cat\# P8341, Sigma-Aldrich). After brief centrifugation to remove debris, the total protein concentration in supernatants was determined, using Bio-Rad (Hercules, CA, United States) DC protein assay reagents (Cat\# 500-0006, Bio-Rad). After $10 \mathrm{~min}$ of heating at $75^{\circ} \mathrm{C}$ in loading buffer (Cat\# NP 0007, Invitrogen) supplemented with reducing reagent (Cat\# NP 0004, Invitrogen), $25 \mu \mathrm{g}$ of protein were loaded from each sample. The protein was separated in $4-12 \% \mathrm{NuPAGE}^{\circledR}$ Bis-Tris gradient mini gels (Cat\# NP 0321BOX, Invitrogen), and transferred onto a PVDF membrane, using a Bio-Rad transblot apparatus. The membranes were blocked in 5\% non-fat dry milk in TRIS-buffered saline (TBS) for $1 \mathrm{~h}$ at RT. Membranes were probed with an antiAkt-T308 antibody (Cat\# 2965, Cell Signaling) diluted 1:1000 at RT for $1 \mathrm{~h}$, or anti-Actin (Cat\# MAB1501, Chemicon) diluted $1: 10,000$ at $4^{\circ} \mathrm{C}$ overnight. After washes with TBS, the blots were incubated with secondary antibodies IRDye $800 \mathrm{CW}$ goat antirabbit IgG (Cat\# 926-32211, LI-COR) or IRDye 680RD goat anti-mouse IgG (Cat\# 926-68070, LI-COR) at 1: 20,000 for $1 \mathrm{~h}$ at RT in the dark. The blots were washed three times with TBS, $10 \mathrm{~min}$ each, scanned and quantified with an Odyssey CLx (LI-COR), and processed with Adobe Photoshop (San Jose, CA, United States).

\section{Immunohistochemistry}

To test whether in vivo ChABC treatment can digest CSPGs in lamprey spinal cord. We applied $\mathrm{ChABC}$ onto the intact spinal cord at the level of the fifth gill, and sacrificed the lampreys after $4 \mathrm{~h}$ recovery. We also applied ChABC in vivo immediately after TX at the fifth gill, and allowed the lampreys to survive for 2 weeks before being sacrificed. The spinal cords between the second gill and $5 \mathrm{~mm}$ caudal to the seventh gill were fixed, dehydrated and embedded in paraffin. Sagittal $10 \mu \mathrm{m}$ thick sections were mounted onto glass slides. After de-paraffinization and rehydration, sections underwent the chromogen reaction. Endogenous horseradish peroxidase (HRP) was quenched with $5 \% \mathrm{H}_{2} \mathrm{O}_{2}$ in methanol for $30 \mathrm{~min}$ at RT. Sections then were washed three times with PBS, $10 \mathrm{~min}$ each. All the sections were incubated with $10 \%$ fetal bovine serum (FBS) $/ 0.2 \%$ Tween20/PBS for $1 \mathrm{~h}$ at RT and then incubated with primary antibody 2 B6 (Cat\# 270432, Seikagaku Biobusiness Corporation) at a dilution of $1: 200$ in $10 \%$ FBS/0.2\% Tween-20/PBS overnight at $4^{\circ} \mathrm{C}$. Sections were washed three times with PBS, $10 \mathrm{~min}$ each, and then incubated with either goat anti-mouse IgG-conjugated HRP (Cat\# SC-2005, Santa Cruz) at 1:200 or donkey anti-mouse IgG-conjugated Alexa Fluor ${ }^{\circledR} 594$ (Cat\# R37121, Thermo Fisher Scientific) at 1:200, in 10\% FBS/0.2\% Tween-20/PBS for $1 \mathrm{~h}$ at
RT. All sections were washed three times with PBS, 10 min each. After incubation in HRP-secondary antibody, sections were washed with PBS and the chromogen reaction was performed with diamino benzidine (DAB). If sections were incubated in fluorescently tagged secondary antibody, the sections were washed with PBS and mounted with Fluoromount-G (Cat\# 010001, SouthernBiotech). Brightfield or fluorescence images were captured with a Nikon $80 \mathrm{i}$ microscope.

To measure levels of Akt phosphorylation at threonine 308 in individual identified neurons, after fixation, dehydration, and paraffin embedding, $10 \mu \mathrm{m}$ thick paraffin sections were mounted onto glass slides for further investigation. All of the brain sections were de-paraffinized, rehydrated, and washed in PBS. Antigen retrieval was performed as follows: sections were immersed in the sodium citrate buffer (10 mM sodium citrate, $\mathrm{pH}$ 6.0). The buffer was boiled for $20 \mathrm{~min}$, and the sections allowed to cool for $20 \mathrm{~min}$ at RT. Sections were rinsed in PBS twice for 5 min each time. All the sections were blocked with 10\% FBS/0.2\% Tween-20/PBS for $1 \mathrm{~h}$ at RT, and incubated overnight with primary antibody antiAkt-T308 (Cat\# 2965, Cell Signaling) at 1:1000 in 10\% FBS/0.2\% Tween-20/PBS at $4^{\circ} \mathrm{C}$. Sections were washed three times with PBS, 10 min each and then incubated with donkey anti-rabbit Alexa Fluor ${ }^{\circledR} 594$ (Cat\# A21207, Thermo Fisher Scientific) at 1:200, in blocking buffer for $1 \mathrm{~h}$ at RT. After incubation in secondary antibody, sections were washed three times with PBS, 10 min each time. Autofluorescence was carefully quenched with the TrueView kit (Cat\# SP-8400, Vector TrueView). Sections were mounted with Fluoromount-G (Cat\# 0100-01, SouthernBiotech). DAF-488 and pAkt-308 fluorescence signaling were captured with a Nikon $80 \mathrm{i}$ microscope under consistent parameters to allow quantification of pAkt-308 fluorescence. All brain sections with identified neurons were collected and quantified with NISElements AR 3.10. For each brain section, all the identified neurons that were filled with DAF-488 were outlined and pAkt308 intensity was measured. Background fluorescence intensity was measured by outlining the area adjacent to the brain. The fluorescence intensity for each section was calculated as follows: the background fluorescence intensity was subtracted from the mean fluorescence intensity within identified neurons in the same section. For each animal, the average fluorescence intensity from all the sections was calculated. Then an overall mean fluorescence was calculated as the mean of all these average intensities.

\section{Statistical Analysis}

Data sets were analyzed with InStat software (GraphPad), normally distributed data were further analyzed by InStat to determine if standard deviations (SD) were equal. An unpaired $t$-test was used for comparison between data sets with equal SD. For western blots, to avoid between-blot variation, all the groups were normalized against loading controls (actin). Then the experimental groups were compared with their respective normalized control groups, whose relative densities were assigned a value of 1 . A paired $t$-test was performed to compare the density difference between groups. The effects of $\mathrm{ChABC}$ on apoptosis signaling was determined for individual identified neurons, by comparing FLICA labeling for the same RS 
neurons in control and ChABC-treated groups, using the paired $t$-test. For normally distributed data sets requiring multiple group comparisons, we used one-way analysis of variance (ANOVA) followed by the Tukey's multiple comparisons test. For correlation analysis, we used the Pearson correlation test. All values were expressed as mean \pm SEM.

\section{RESULTS}

\section{ChABC Digests CSPGs in Lamprey Spinal Cord}

Chondroitinase $\mathrm{ABC}$ was applied in vivo onto the lamprey spinal cord at the level of the 5th gill, with or without TX (detail in section "Materials and Methods"). After ChABC application, there was strong staining of CSPG stumps (2B6) in control and TXed spinal cords (Figures 1A,C), suggesting that $\mathrm{ChABC}$ had digested the sugar chains of CSPGs, and thereby exposed the stumps (2B6). $\mathrm{H}_{2} \mathrm{O}_{2}$ was used to eradicate endogenous HRP activity during IHC staining and chromogenic reaction. The results were confirmed by immunofluorescence staining with the $2 \mathrm{~B} 6$ antibody, to show the distribution of CSPG stumps after ChABC treatment in control and TXed spinal cords. Figures 1B,D show strong immunofluorescence in the same area as indicated in Figures 1A,C. These results confirmed that $\mathrm{ChABC}$ application in vivo can digest endogenous CSPGs effectively in lamprey CNS, either in control lamprey, or after spinal cord TX.

\section{Digestion of CSPGs With ChABC at the Site of Injury Inhibits Axon Retraction in the Proximal (Rostral) Stump at Early Stage and Promotes Axon Regeneration at Late Stage}

At the early stage after SCI in the lamprey, transected axons can form axon tips which undergo retraction first and then begin to grow forward toward the TX site, reaching the injury scar by 4 weeks. These axon tips can be imaged in vivo, and thereby can be assessed by measuring the distance between the axon tips and TX site (Jin et al., 2009; Hu et al., 2017). We transected the spinal cords at the level of the 5 th gill and applied either enzyme buffer or ChABC with DTMR to the TX site for 2 weeks (Figure $2 \mathrm{~A}$ ). The whole-mounted free spinal cords were carefully dissected out. The images of axon tips were taken by fluorescence microscopy, so that the distances between individual axon tips (arrowheads in Figures 2B,C) and the TX sites (dashed lines in Figures $\mathbf{2 B}, \mathbf{2 C}$ ) could be measured. The mean distances between the axon tips and TX sites in ChABC-treated spinal cords ( $683 \pm 62 \mu \mathrm{m} ; n=30$ from five lampreys) were shorter than that in enzyme buffer-treated ones $(1026.03 \pm 106 \mu \mathrm{m} ; n=31$ from five lampreys, $p<0.01$ ) at 2 weeks post-TX (Figure 2D). This result suggested that ChABC digestion of CSPGs inhibits the retraction of transected axons, either by reducing the retraction distance or by promoting the early start of axon regrowth.

We also assessed the axon regeneration after ChABC treatment at late stage (10 weeks post-TX). A first TX was performed at the fifth gill and Gelfoam was applied with either enzyme buffer (control) or ChABC in enzyme buffer. After 10 weeks, a 2nd TX was performed $5 \mathrm{~mm}$ caudal to the first TX, and DTMR applied as a retrograde tracer to both groups (control vs. ChABC) (Figure 2E). After 1 more week, the retrogradely labeled RS neurons in the brain were counted carefully and these were considered neurons whose axons had undergone true regeneration (not collateral sprouting) at least $5 \mathrm{~mm}$ beyond the TX (Figure 2E). The total number of DTMR-positive identified RS neurons was greatly increased at 10 weeks post-TX with ChABC treatment compared to control enzyme buffer treatment (Figures $\mathbf{2 F}-\mathbf{H}$ ). We organized all of the identified RS neurons by their locations in brain $\left(M_{1}-M_{4}\right.$ are in mesencephalon; $\mathrm{I}_{1-} \mathrm{I}_{6}$ are in anterior rhombencephalon; $\mathrm{B}_{1-} \mathrm{B}_{6}$, Mth and $\mathrm{mth}^{\prime}$ are in the middle rhombencephalon). There were increased numbers of DTMR-labeled identified RS neurons in all three of these regions, among which the RS neurons in the anterior rhombencephalic region showed the most dramatic regeneration (Figure $\mathbf{2 H}$ ). Since individual identified RS neurons have different regeneration probabilities, the effects of ChABC treatment might be different for each of the individual identified RS neurons. We calculated the regeneration probability of individual identified RS neurons after TX with or without ChABC treatment (Figure 2I). Except for $\mathrm{M}_{4}, \mathrm{I}_{2}, \mathrm{~B}_{2}, \mathrm{~B}_{5}$, and $\mathrm{mth}^{\prime}$, all the other identified RS neurons benefited from ChABC treatment. The poorly regenerating neurons $\mathrm{I}_{1}, \mathrm{Mth}, \mathrm{B}_{3}$, and $\mathrm{B}_{4}$ and the good regenerators $\mathrm{M}_{1}, \mathrm{I}_{3}, \mathrm{I}_{4}$, and $\mathrm{I}_{5}$, all had greater regeneration probabilities with $\mathrm{ChABC}$ treatment. Thus, in lamprey, ChABC promotes true axon regeneration after spinal cord TX.

\section{Digestion of CSPGs With ChABC Reduces Retrograde Neuronal Apoptosis Signaling}

Fluorochrome-labeled inhibitor of caspase activity staining was performed on brains at 1, 2, 4, 8, and 11 weeks after spinal cord TX. Compared to uninjured controls (Figure 3A), the number of all RS neurons showing polycaspases activity increased significantly at 2, 4, 8, and 11 weeks post-TX (Figures 3D,F,H,J,L). ChABC did not affect the number of polycaspases positive (FLICA+) RS neurons at 1 week post-TX (Figure 3B vs. C, Figure 3L), but reduced it significantly at 2 weeks (Figure 3D vs. E, Figure 3L). This reduction effect disappeared at 4 weeks (Figure 3F vs. G, Figure 3L) and 8 weeks (Figure $3 \mathrm{H}$ vs. I, Figure 3L). Interestingly, at 11 weeks after TX, the number of FLICA+ neurons decreased again in ChABC-treated lampreys (Figure 3J vs. K, Figure 3L), suggesting that the ChABC effects on retrograde neuronal death outlast the caspase activation. This is consistent with the effects of ChABC to increase axon regeneration at 10 weeks post-TX (Figures $2 \mathrm{E}-\mathrm{I}$ ). It has been reported that caspases are not involved only in apoptosis, but also play critical roles in multiple cellular processes in the nervous system (Hu et al., 2013). The polycaspases FLICA reagent that we used in this study labels all active caspases and does not specifically target any single caspase. This may explain why ChABC did not affect the number of poly-caspase positive neurons at 4 and 8 weeks post-TX. To clear up this ambiguity, 

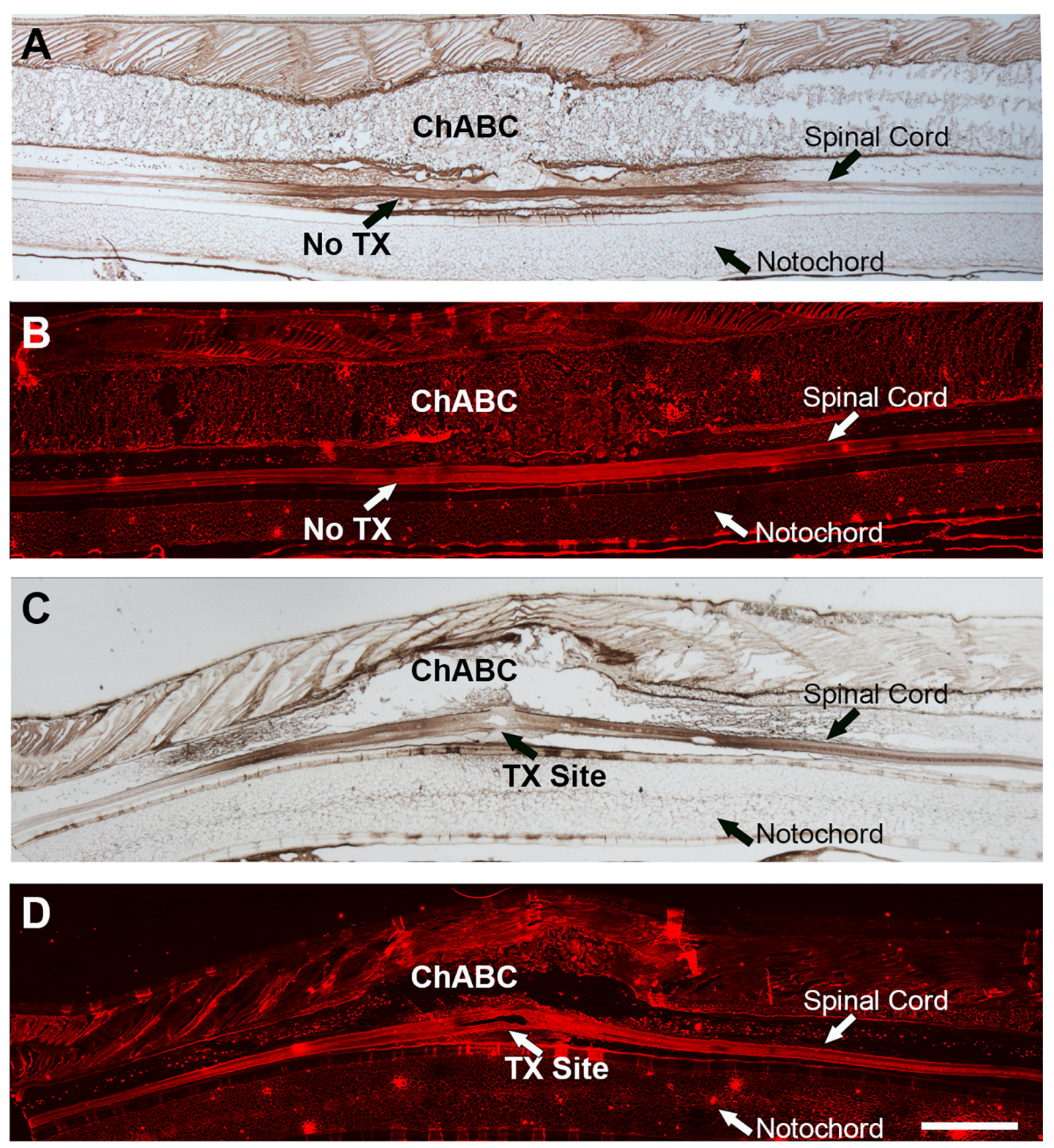

FIGURE 1 | ChABC treatment of lamprey spinal cord after transection. Stumps of digested CSPGs were labeled with mAb $2 B 6$ and imaged in sagittal sections of spinal cord by colorimetric immunohistochemistry (A,C) or Alexa Fluor-594 immunofluorescence (B,D). A,B are intact lamprey spinal cord treated with ChABC. C,D are sections of a spinal cord that had been transected and treated with ChABC 2 weeks previously. Scale: $1 \mathrm{~mm}$.

caspase 3-specific FLICA was used (Figure 4), which targets only neurons undergoing apoptosis. ChABC significantly reduced the number of caspase 3-positive RS neurons at 4 weeks post-TX (Figure 4). Thus ChABC treatment had a beneficial effect by reducing retrograde apoptosis after SCI.

\section{ChABC Inhibits PTPo mRNA Expression}

Because PTP $\sigma$ is thought to be a receptor for CSPGs, we determined the effect of digesting CSPGs with ChABC on the expression of PTP $\sigma$. ISH was used to detect the level of $\mathrm{PTP} \sigma$ mRNA expression in identified RS neurons at 2 and 8 weeks post-TX. In this set of experiments, we identified the
18 pairs of RS neurons by their specific anatomical locations and unique cell body morphologies. We found that ChABC not only reduced polycaspases activation at 2 weeks post-TX (Figure 5A vs. C), but also reduced the number of neurons expressing PTP $\sigma$ mRNA (Figure $5 \mathrm{~B}$ vs. D, Figure $5 \mathbf{K}$ ). РTP $\sigma$ mRNA expression correlated with polycaspases FLICA labeling in RS neurons at 2 weeks post-TX, both without $(r=0.8886$, $p<0.001)$ and with ChABC treatment $(r=0.8729, p<0.001)$ (Figure 5E). Although the effect of $\mathrm{ChABC}$ on polycaspases activity had disappeared by 8 weeks post-TX (Figure 5F vs. $\mathbf{H})$, the number of neurons expressing PTP $\sigma$ mRNA remained reduced (Figure 5G vs. I, Figure 5K). The correlations persisted 

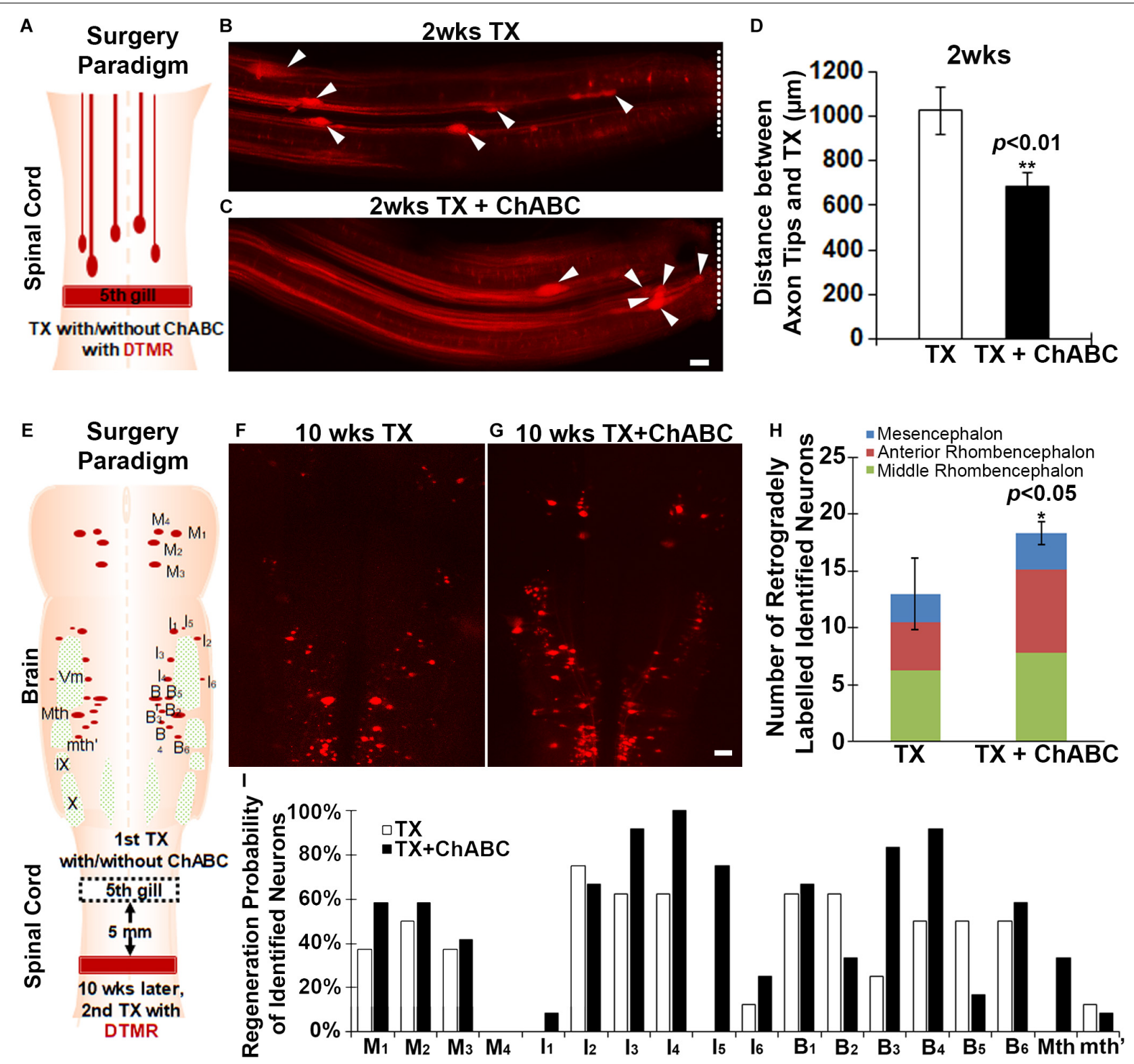

FIGURE 2 | ChABC slows initial axon retraction in vivo at 2 weeks and promotes axon regeneration in vivo at 10 weeks post-TX. (A) Experimental design for detecting the axon tips at 2 weeks post-TX. A solution of DTMR and either ChABC or control enzyme buffer was applied to a fresh spinal cord TX. (B,C) Axon tips (arrowheads) in the lamprey spinal cord back-filled with DTMR at 2 weeks post-TX (TX sites are labeled by dashed lines). (D) Mean distances of axon tips from the center of the TX site (dashed line). ${ }^{* *} p<0.01, n=$ number of axon tips. (E) Experimental design for detecting the neurons whose axons have regenerated by 10 weeks post-TX. The TX site was treated with either control enzyme buffer (F), or ChABC (G) at the time of injury. Ten weeks later, DTMR was applied to a second TX $5 \mathrm{~mm}$ caudal to the first, and 1 week allowed for retrograde transport of the dye. (H) A graph showing an increased number of retrogradely labeled neurons in brains of animals treated with ChABC compared to controls. (I) The probability for each identified neuron that its axon will have regenerated at 10 weeks post-TX. ${ }^{*} p<0.05, n=5$ lampreys per group. Error bars: SEM. Scale bar: $100 \mu \mathrm{m}$.

at 8 weeks post-TX (Figure 5J; $r=0.8985, p<0.0001$, and $r=0.7145, p<0.001$, respectively).

\section{ChABC Increases Akt Activation}

Akt is an important downstream target signaling molecule for CSPG receptors in neurons in vitro (Fisher et al., 2011), and Akt activity is thought to be a signaling molecule that promotes axon regeneration after SCI. Therefore, the effect of ChABC on the activation status of Akt was investigated, using phosphorylation at threonine 308 (pAkt-308) as an indicator. ChABC treatment after SCI increased Akt phosphorylation levels in the brain at 1,2, 4 , and 8 weeks post-TX (Figures 6A,B). This long-term activation of Akt after ChABC treatment is consistent with its enhancement of long-term axon regeneration described above (Figures 2E-I).

Since Akt is expressed widely in both neurons and glial cells, the western blots cannot specify whether Akt activation 

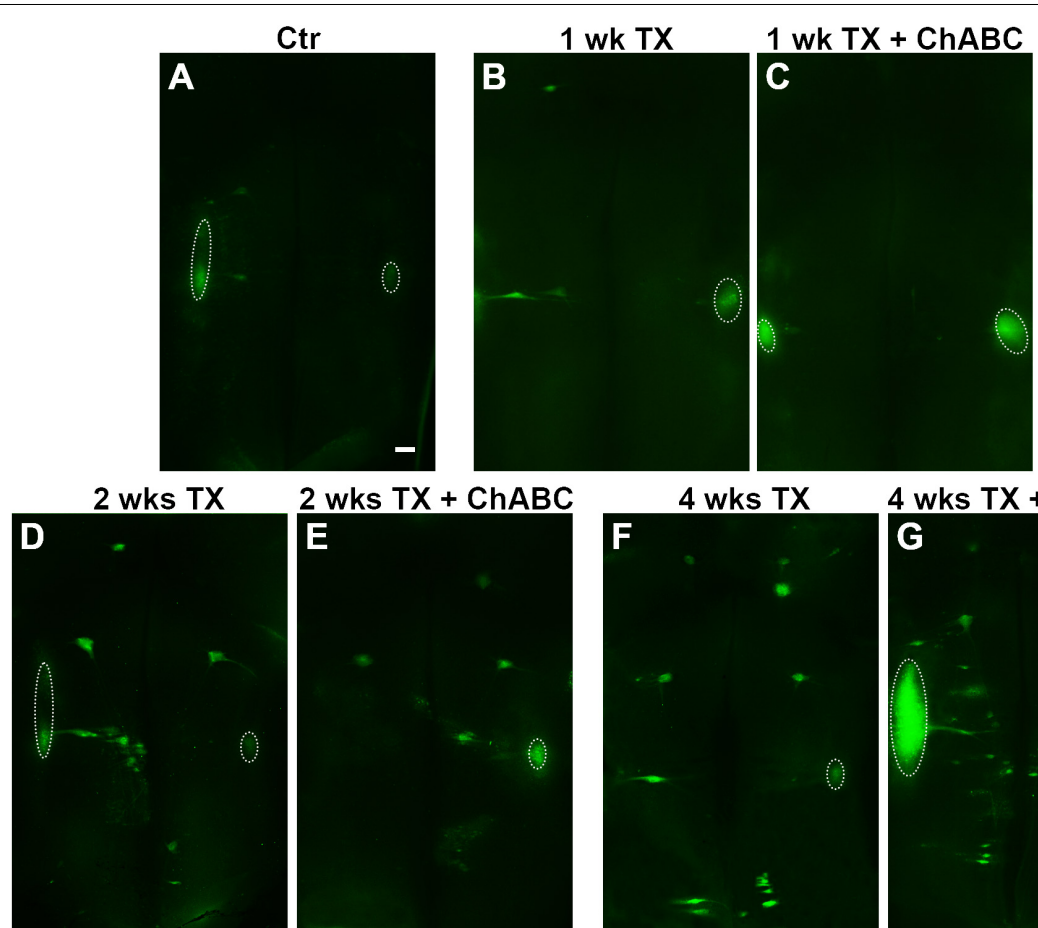

2 wks TX + ChABC

4 wks TX

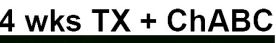

8 wks TX
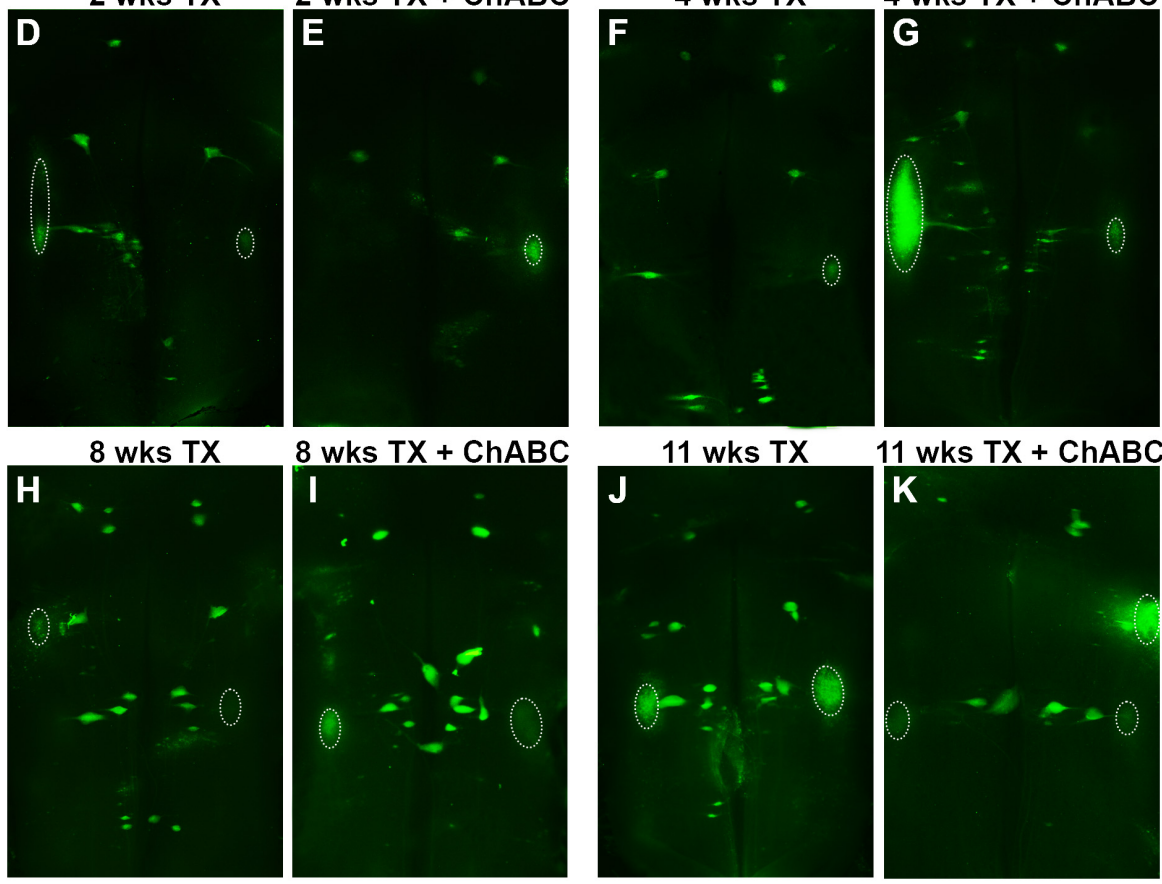

8 wks TX + ChABC

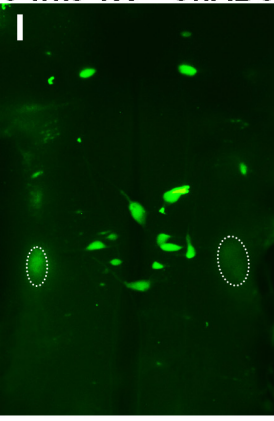

11 wks TX
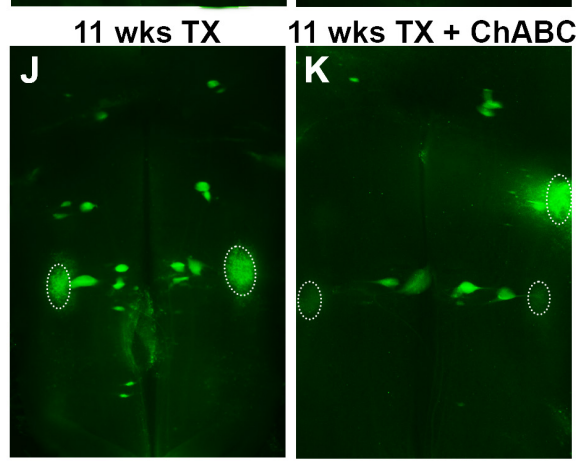

$\mathbf{K}$

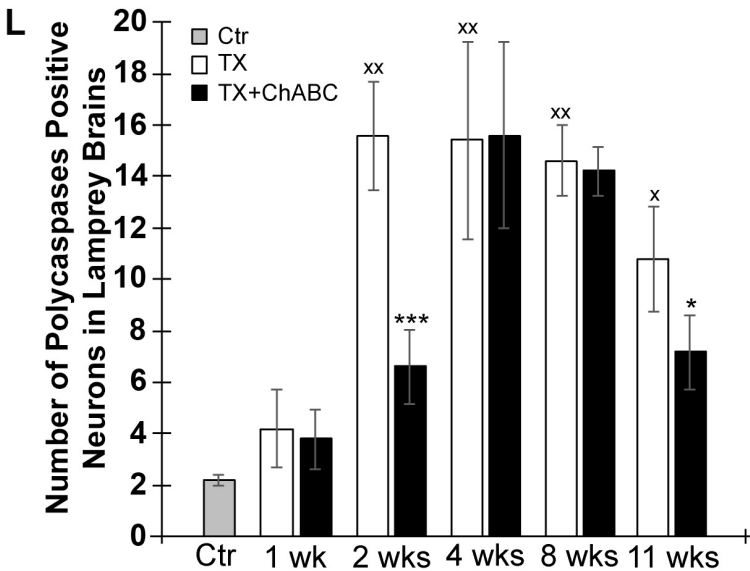

FIGURE 3 | Polycaspase activation in RS neurons is suppressed by ChABC. Spinal cords were transected at the 5th gill with/without ChABC, and allowed to recover for 1-11 weeks. (A) FLICA on control brain (without TX). ChABC did not affect the number of polycaspase positive (polycaspase FLICA) RS neurons at 1 week post-TX (B vs. C), but reduced it significantly at 2 weeks (D vs. E) and at 11 weeks (J vs. $\left.\mathbf{K},{ }^{*} p<0.05\right)$, but not at 4 (F vs. G) or 8 weeks (H vs. I). (L) A graph showing the number of polycaspase positive identified RS neurons per brain at 1, 2, 4, 8, and 11 weeks post-TX with or without ChABC. Polycaspase positive RS neurons increased greatly at 2, 4, 8, and 11 weeks comparing to control brain $\left({ }^{x} p<0.05,{ }^{x x} p<0.01\right)$; ChABC reduced polycaspase positive RS neurons at 2 $\left({ }^{\star \star \star} p<0.001\right)$ and 11 weeks $\left({ }^{*} p<0.05\right)$. The large fluorescent accumulations (outlined by circles) seen laterally are out of focus cranial motor nuclei, which are axotomized close to their perikaryal at the time of live dissection, so their neurons rapidly turn caspase-positive. $n=5$ lampreys per group. Error bars: SEM. Scale bar: $200 \mu \mathrm{m}$. 

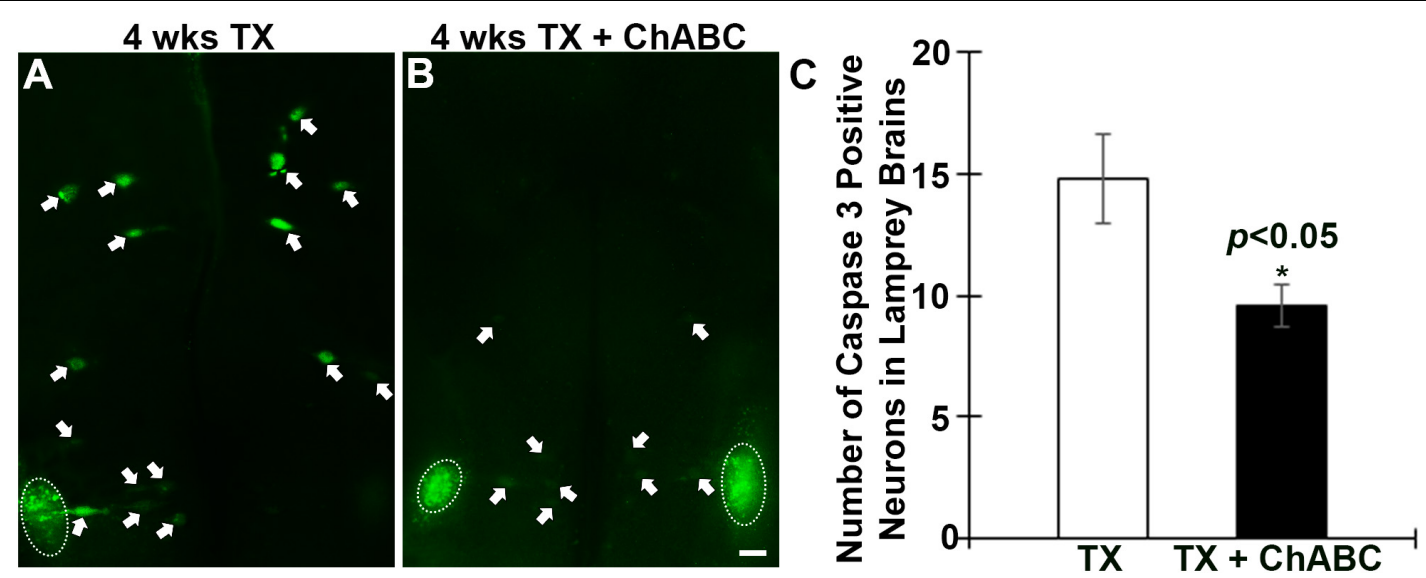

FIGURE 4 | Active caspase 3 in RS neurons is reduced by ChABC at 4 weeks post-TX. Spinal cords were transected at the 5th gill with or without ChABC, and allowed to recover for 4 weeks. ChABC reduced the number of caspase 3-positive RS neurons at 4 week post-TX significantly (A vs. B). (C) A graph showing the number of caspase 3-positive identified RS neurons per brain at 4 weeks post-TX with or without ChABC. Arrows point to the active caspase 3 positive RS neurons, and circles outline cranial motor nuclei, whose neurons rapidly become caspase positive when they are axotomized during brain dissection. ${ }^{\star} p<0.05, n=5$ lampreys per group. Error bars: SEM. Scale bar: $100 \mu \mathrm{m}$.

occurs specifically in axotomized neurons (Figure 6). Therefore, we determined the activation status of Akt in individually identified RS neurons after ChABC treatment. RS neurons were retrogradely labeled by DAF-488 applied to an acute spinal cord TX, with or without ChABC treatment. The glial cells would not be labeled because they lack the long axons that would allow for retrograde labeling. Lampreys were sacrificed at 2, 4, and 8 weeks post-TX, and the brains fixed and processed for paraffin sectioning. The expression levels of pAkt-308 in the individual RS neurons were determined by immunofluorescence staining. The individual identified neurons were recognized with the retrogradely labeled DAF488 (green, Figures 7A,C,F,H,K,M), and then by pAkt308 immunofluorescence (red, Figures 7B,D,G,I,L,N). Akt phosphorylation intensity was quantified in the DAF-488 retrogradely labeled neurons. Thus, we included only neurons that were filled with the retrograde dye DAF-488, i.e., RS neurons. At 2, 4, and 8 weeks post-TX, the fluorescence intensity in identified RS neurons of ChABC-treated lampreys was approximately $15 \%$ greater than in those treated with control enzyme buffer (Figures $\mathbf{7 A - E}, \mathbf{F}-\mathbf{J}$, and $\mathbf{K}-\mathbf{O}$ ), respectively; $p<0.01)$. Thus digestion of CSPGs with ChABC significantly increased the level of activation of the pro-growth signaling molecule Akt in the individually identified RS neurons of the lamprey brain.

\section{DISCUSSION}

We investigated the effects of removing the polysaccharide side chains of CSPGs with ChABC on axon regeneration of RS neurons, and on retrograde apoptosis of their cell bodies in the brainstem, after SCI in the lamprey. IHC of CSPG stubs confirmed the digestion of CSPGs by application of ChABC in vivo. FLICA labeling was used to quantify caspase activation, i.e., apoptosis signaling, which previously was shown to be increased after spinal cord TX, primarily in "bad-regenerating" identified RS neurons in lamprey brain (Barreiro-Iglesias and Shifman, 2012; Hu et al., 2013).

\section{Digestion of CSPGs With ChABC Reduces Retrograde Apoptotic Signaling}

Caspases are a family of cysteine proteases that are found in a wide range of animals, from worms to humans, and are involved in apoptosis. The poly-caspase FLICA we used in the present study detects most caspases, including caspase 1 , $3,4,5,6,7,8$, and 9 . The number of identified RS neurons containing activated polycaspases increased significantly at 2 , 4 , and 8 weeks post-TX, compared to controls. These findings are consistent with our previous findings that neurons known to be bad regenerators eventually die by a very delayed form of TUNEL-positive apoptosis (Shifman et al., 2008). CSPGs, which are normal constituents of the perineuronal nets in CNS (Bruckner et al., 2000; Deepa et al., 2006), are greatly elevated after SCI (Bradbury et al., 2002; Zhang et al., 2014). We successfully digested the elevated CSPGs by ChABC at the TX site in the spinal cord. Digestion of CSPGs significantly reduced the number of polycaspase positive RS neurons at 2 and 11 weeks post-TX, but showed no beneficial effects at 4 and 8 weeks post-TX. However, because in addition to their role in apoptosis, many caspases also play critical roles in multiple physiological processes in the nervous system, such as dendritic remodeling (Kuo et al., 2006; Williams et al., 2006) and synaptic plasticity (Lu et al., 2006; Li et al., 2010). Even caspase- 3 has been found in some cases to participate in nonapoptotic functions in CNS (D'Amelio et al., 2010), but caspase3 is an executioner caspase, which is more directly involved in apoptosis than are the upstream caspases. Therefore, we used caspase 3-specific FLICA to selectively target RS neurons undergoing apoptosis. ChABC significantly reduced the number 

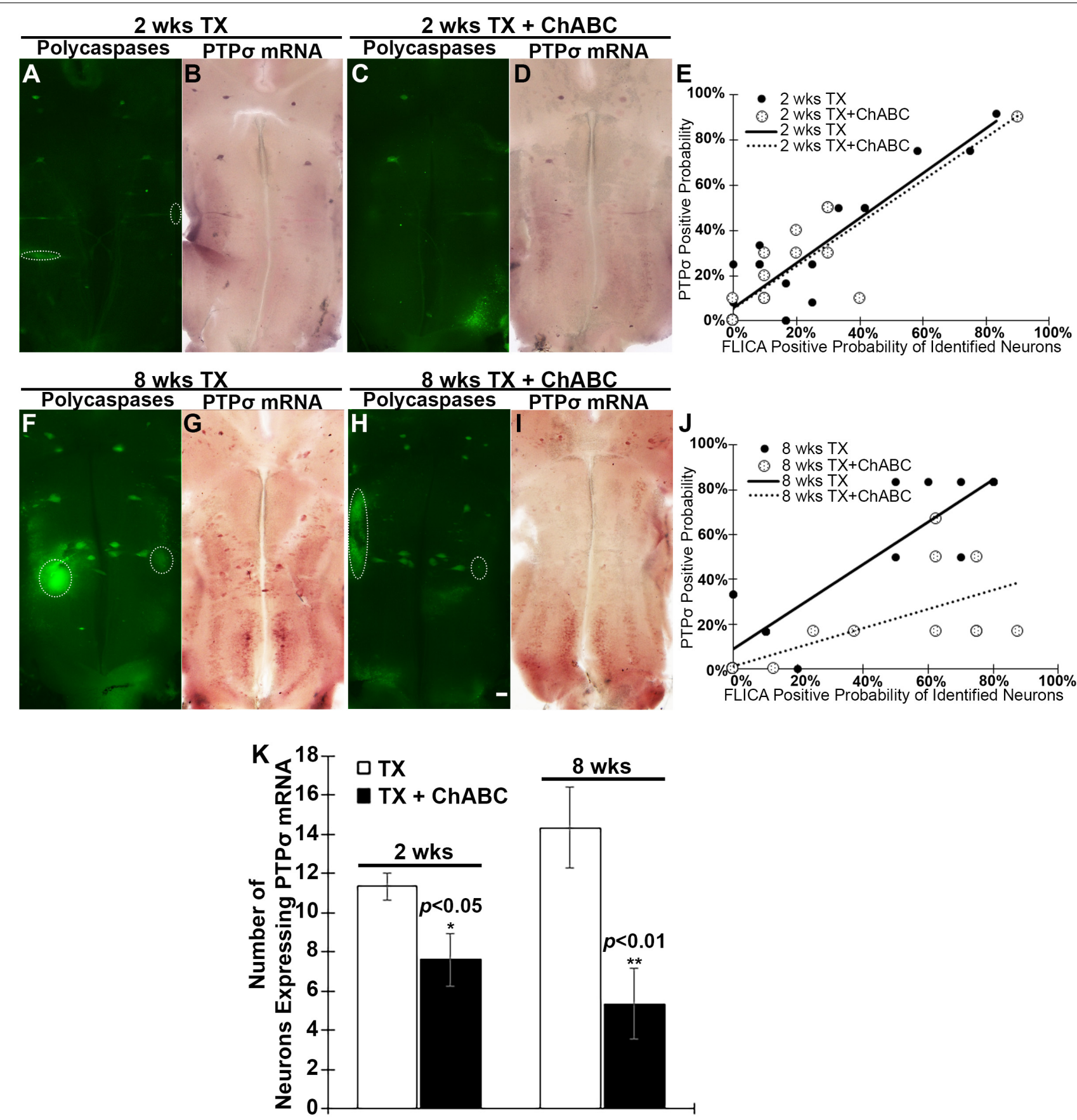

FIGURE 5 | ChABC reduces PTPo mRNA expression in brain at 2 and 8 weeks post-transection. At 2 weeks post-TX, ChABC reduced caspase activation (A vs. C), and also reduced the number of neurons expressing PTP $\sigma$ mRNA (B vs. D). (E) PTP $\sigma$ mRNA expression correlated strongly with FLICA in RS neurons at 2 weeks post-TX, both with control enzyme buffer treatment $(r=0.8886, p<0.001)$ and with ChABC $(r=0.8729, p<0.001)$. The effect of ChABC on caspase activity disappeared at 8 weeks post-TX (F vs. $\mathbf{H})$, but the number of neurons expressing PTP $\sigma$ mRNA was still reduced (G vs. I). (J) At 8 weeks post-TX, there was a strong correlation between PTP $\sigma$ mRNA and FLICA in identified RS neurons, both with control enzyme buffer $(r=0.8985, p<0.001)$ and with ChABC treatment $(r=0.7145, p<0.001)$. (K) The number of identified RS neurons expressing PTP $\sigma$ mRNA was reduced greatly by ChABC treatment at 2 and 8 weeks post-TX. Circles outline cranial motor nuclei, whose neurons rapidly become caspase positive when they are axotomized during brain dissection. ${ }^{*} p<0.05,{ }^{* *} p<0.01, n=5$ lampreys per group. Error bars: SEM. Scale bar: $200 \mu \mathrm{m}$.

of those RS neurons at 4 weeks post-TX. Based on the caspase 3 results at 4 weeks, it is reasonable to conclude that lack of reduction in polycaspace activity does not exclude reduced caspase 3 activity after ChABC treatment at 8 weeks, as well. We could have repeated the caspase 3 -specific assays at 8 weeks, but felt that the 4 -week result was proof of principle. Thus, 


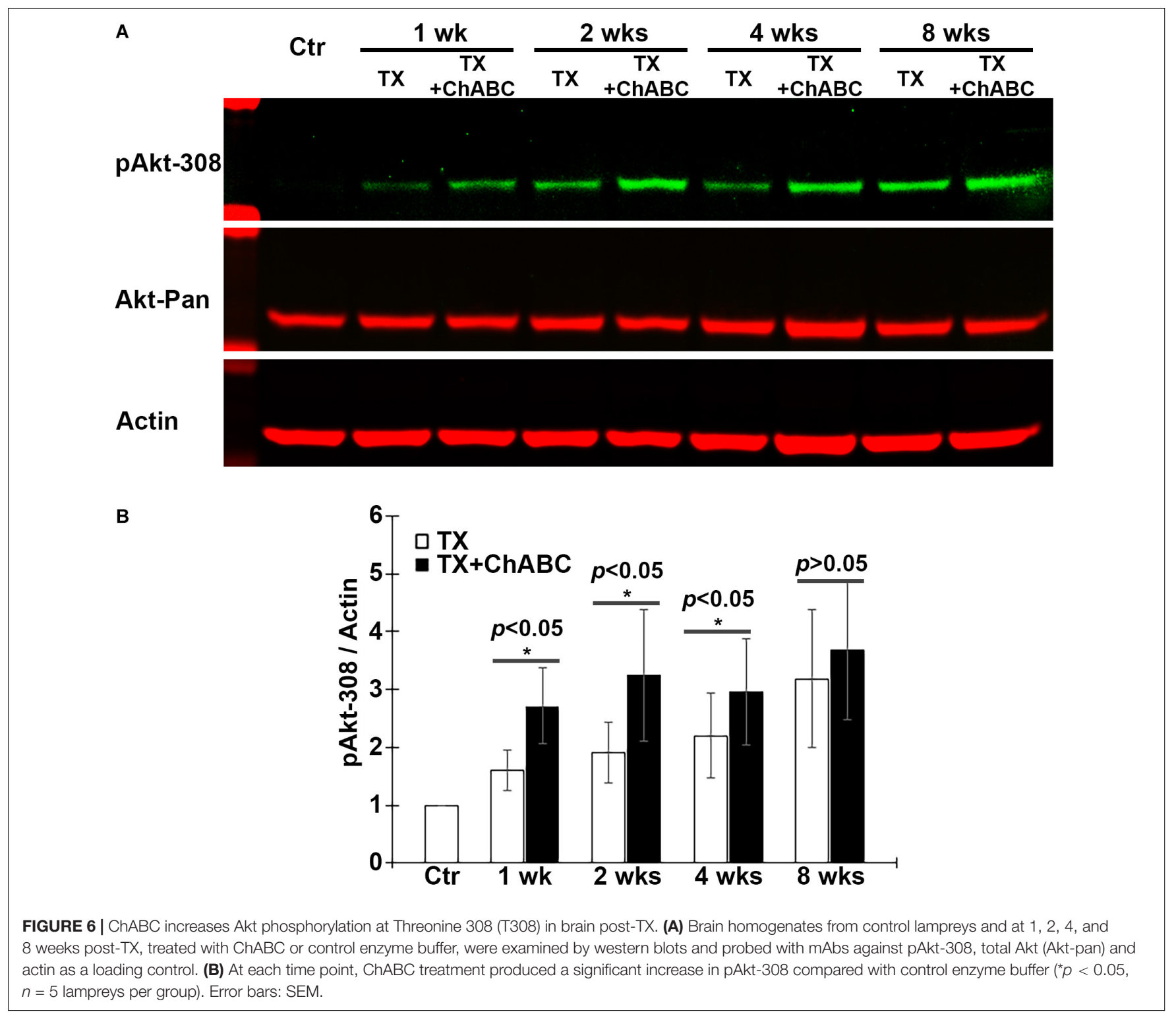

ChABC treatment after SCI can greatly decrease retrograde apoptotic signaling.

\section{Digestion of CSPGs With ChABC Promotes Axon Regeneration}

Chondroitinase $A B C$ treatment has beneficial effects over the transected axons. At 2 weeks post-TX, ChABC treatment reduced the distance between the axon tips and TX sites. Moreover, it greatly increased the number of individually identified RS neurons that could be labeled retrogradely from $5 \mathrm{~mm}$ caudal to a spinal cord TX at 10 weeks post-TX, indicating that ChABC treatment promotes axon regeneration after SCI. The beneficial effects of ChABC on identified RS neurons were seen in the mesencephalon, and the anterior and middle rhombencephalon. The ChABC treatment also changed the probabilities of regenerating for individual identified RS neurons, which included both bad regenerators and good regenerators. These findings suggest that $\mathrm{ChABC}$ treatment is generally beneficial for axon regeneration of these identified RS neurons. This might be due to retrograde signaling in the injured axons due to their interaction with CSPGs secreted at the site of injury. Previous reports in mammals suggested that in vivo, ChABC application in the intact spinal cord can induce axon sprouting (Galtrey et al., 2007). In rats with bilateral dorsal column lesions, $\mathrm{ChABC}$ treatment promoted growth of spinal axons and functional recovery (Bradbury et al., 2002). Digestion of the CSPGs with ChABC enhanced sensory recovery after unilateral cervical rhizotomy at $\mathrm{C} 5, \mathrm{C} 6, \mathrm{C} 8$, and $\mathrm{T} 1$, sparing $\mathrm{C}$. This was accomplished via reorganization of intact $\mathrm{C} 7$ primary afferent terminals - not by regeneration of severed afferents back into the spinal cord (Cafferty et al., 2008). Unilateral pyramidotomy in spinal cord-injured mice elicited robust sprouting of the uninjured CST, with numerous axons observed crossing the 


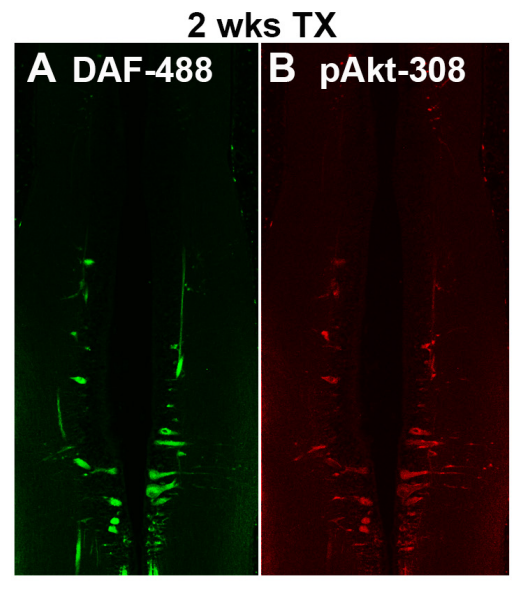

4 wks TX

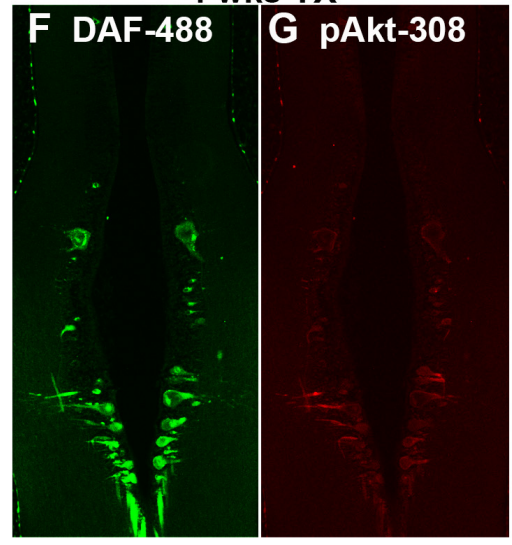

8 wks TX

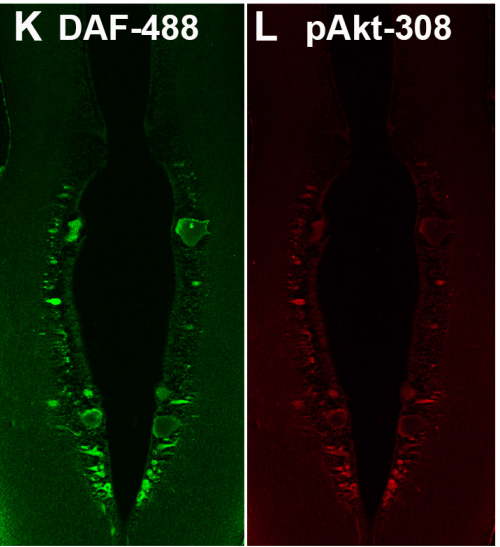

2 wks TX + ChABC

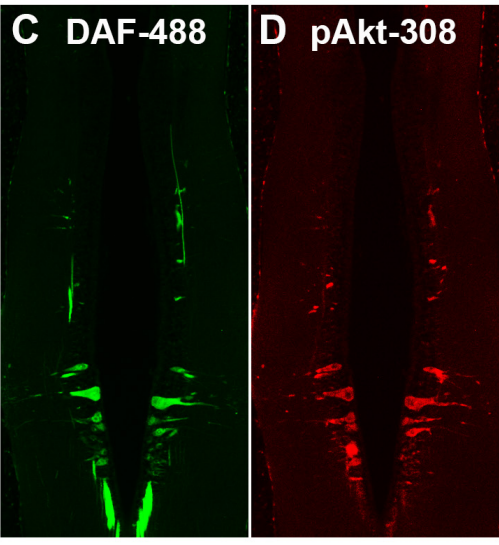

4 wks TX + ChABC

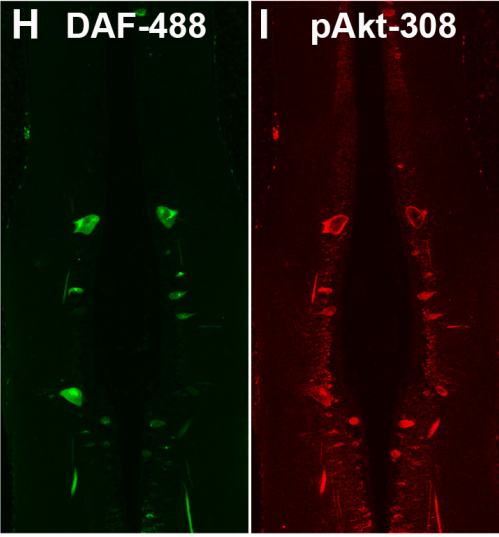

8 wks TX + ChABC

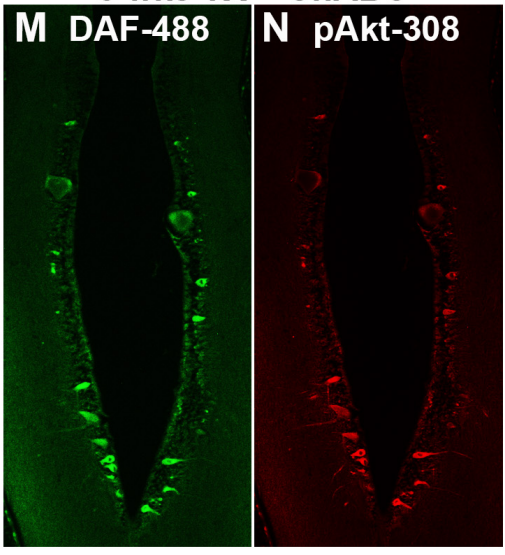

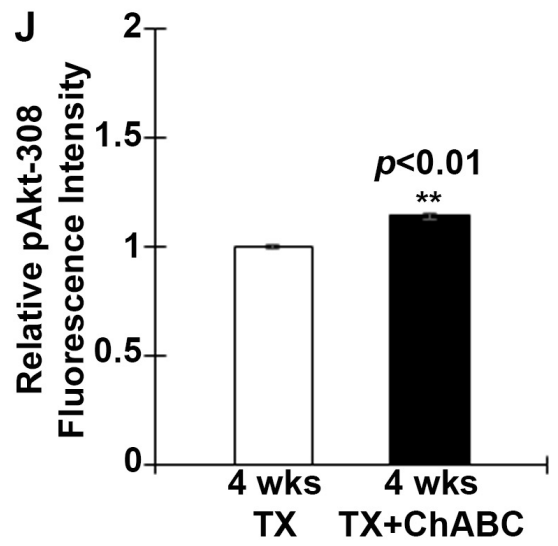
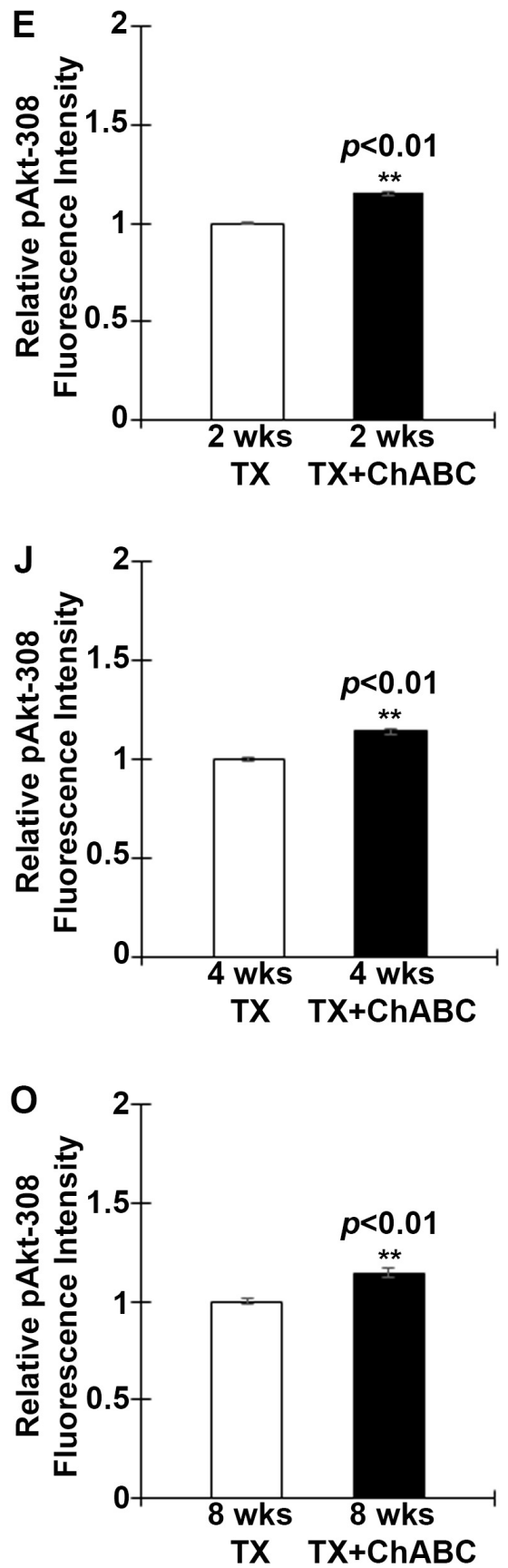

FIGURE 7 | ChABC treatment increases the axotomy-induced activation of Akt in identified RS neurons. RS neurons were retrogradely labeled with DAF-488 and treated with ChABC or control enzyme buffer. Horizontal paraffin sections were prepared and imaged at 2 weeks post-TX (green, A and C). Sections were immunostained for pAkt-308 (Red, B and D). There was a small but statistically significant increase in pAkt-308 fluorescence intensity in identified RS neurons of the ChABC-treated group, relative to the control buffer-treated animals, whose mean intensity was defined as 1 (E; ${ }^{* *} p<0.01, n=3$ lampreys per group). This also was true at 4 weeks (F-J; ${ }^{\star \star} p<0.01, n=5$ lampreys per group) and at 8 weeks post-TX $\left(\mathbf{K}-\mathbf{O} ;{ }^{* \star} p<0.01, n=3\right.$ lampreys per group). Error bars: SEM. Scale bar: $100 \mu \mathrm{m}$.

midline in the brainstem and spinal cord, and terminating in denervated gray matter. This was accompanied by restoration of function (Starkey et al., 2012). Taken together, these studies suggest that digestion of CSPGs with ChABC enhances axon sprouting and functional recovery after SCI in mammalian models, but because these models involved partial SCI, it is unclear whether this beneficial effect was due entirely to collateral sprouting by spared axons, or also involves true regeneration 
of injured axons. This ambiguity was eliminated in the current study on lampreys, because we performed complete spinal cord TX. Thus the increased growth of RS axons beyond the lesion was due to true regeneration, and could not be accounted for by compensatory collateral sprouting by spared axons.

\section{Role of PTPo in Retrograde Neuronal Death}

Chondroitinase ABC treatment at the time of SCI significantly inhibited PTP $\sigma$ mRNA expression in the perikarya of the axotomized identified RS neurons, as assessed at 2 and 8 weeks post-TX. This was accompanied by a concomitant reduction in retrograde neuronal apoptotic signaling (polycaspase FLICA), and is consistent with a role for PTP $\sigma$ in retrograde neuronal apoptosis. This was suggested previously by the selective expression of PTP $\sigma$ in "bad regenerator, bad survivor" RS neurons, which became FLICA-positive after SCI (Hu et al., 2013). More intriguingly, Akt activation (pAkt-308) was enhanced in brains after ChABC treatment, as determined by western blotting, and confirmed in individual identified RS neurons by immunostaining.

\section{Role of PTP $\sigma$ in Axon Regeneration}

Two RPTPs, PTP $\sigma$, and LAR, have been identified as transmembrane receptors for CSPGs (Shen et al., 2009; Fisher et al., 2011; Sharma et al., 2012). Genetic disruption of PTP $\sigma$ promoted axon growth into CSPG-rich regions of SCI (Shen et al., 2009). Transgenic deletion of LAR increased growth of descending axons caudal to the lesion and enhanced locomotor recovery after SCI (Xu et al., 2015). This also was true for systemic injection of small peptide inhibitors of LAR (Fisher et al., 2011) and PTP $\sigma$ (Lang et al., 2015). Our group used antisense morpholinos (MOs) to knock down lamprey PTP $\sigma$ in vivo and studied its direct effects on the axon regeneration and retrograde neuronal death after SCI. Unexpectedly, we found that $\mathrm{PTP} \sigma$ knockdown in lamprey reduced axon regeneration and neuronal survival beginning between 10 and 20 weeks after TX (Rodemer et al., 2020). Those results seem to be inconsistent with the putative role of PTP $\sigma$ in mammalian axon regeneration (Lang et al., 2015), and with the correlation between PTP $\sigma$ mRNA and post-TX retrograde apoptotic signaling seen even after $\mathrm{ChABC}$ treatment in current study. In our previous report, the lack of activated caspases in RS neurons, and the long latency after PTP $\sigma$ knockdown in vivo indicated that enhanced supraspinal neuronal death might result from nonapoptotic mechanisms: by incidentally transfected infiltrating immune cells, or trophic deprivation, or autonomous autophagic mechanisms (Rodemer et al., 2020). It also is possible that the redundancy of CSPG receptors mitigated the beneficial effect of in vivo PTP $\sigma$ knockdown. The use of ChABC can avoid the complications or concerns raised with PTP $\sigma$ knockdown in vivo. The ChABC-induced digestion of elevated CSPGs around the TX site restores the CSPG levels in the environment of the injury site to those found in the un-injured spinal cord. This manipulation is easy to perform and does not directly interfere with other physiologically critical molecules in vivo. Thus, ChABC digestion of elevated CSPGs may be beneficial to axon regeneration and neuronal survival in more than one mechanism.

\section{Digestion of CSPGs With ChABC Reduces Expression of PTP $\sigma$}

Previously, we reported that in lamprey CNS, CSPGs were widely distributed in the extracellular matrix, as well as in cell bodies of the gray matter (Zhang et al., 2014). There was increased CSPG expression at the site of injury, which peaked at 2 weeks post-TX and then gradually decreased to control levels by 10 weeks. In lamprey, both PTP $\sigma$ and LAR mRNAs were expressed primarily in neurons whose regeneration capacity is poor (bad regenerators) in both control brains and brains of animals with SCI. Although ISH suggested that both PTP $\sigma$ and LAR were upregulated after spinal cord TX, the effect was not quantitated at that time. The PTP $\sigma$ mRNA-positive identified RS neurons often included $\mathrm{M}_{2}$ and $\mathrm{M}_{3}$ in the mesencephalon, and $I_{1}, I_{2}$, Mauthner (Mth), $B_{1}, B_{3}$, and $B_{4}$ in the rhombencephalon. In the present study, PTP $\sigma$ mRNA also appeared dramatically in some axons with poor-regenerative ability (including Mth, $\mathrm{B}_{3}$, and $\mathrm{I}_{2}$ ), consistent with the pattern of expression observed in the cell bodies in the brainstem. Of special interest, digestion of CSPGs with ChABC applied at the site of injury reduced the number of RS neurons expressing PTP $\sigma$ mRNA, suggesting that the upregulation of PTP $\sigma$ mRNA observed in RS neurons after SCI is due in part to the actions of elevated CSPGs. Although we cannot determine from the present study whether this is due to a direct effect of CSPGs on the injured axons, our findings are consistent with the recent studies. Lang et al. (2015) found that PТР $\sigma$ becomes concentrated in dystrophic stabilized growth cones and LAR has similar elevation pattern. They also observed a large concentration of PTP $\sigma$ in the lesion penumbra following SCI (Lang et al., 2015). Moreover, there is a report suggesting that CSPGs may upregulate PTP $\sigma$ mRNA and protein levels in neural stem cells in vitro (Zhong et al., 2019). Thus, the ChABC digestion of CSPGs affects PTP $\sigma$ expression in lamprey RS neurons, which may reflect a direct action on the injured axon and a retrograde signal to the neuronal perikaryon.

\section{ChABC Activates Akt}

PTEN knockout promotes potent CNS axon regeneration after injury (Park et al., 2008; Liu et al., 2010; Du et al., 2015), and the signaling molecules downstream of PTEN that mediate this effect have been studied extensively (Liu et al., 2011). It has been reported that Akt activation can promote optic nerve axon regeneration and survival of RGC (Yang et al., 2014; Guo et al., 2016). GSK3 $\beta$ plays an indispensable role in mediating Akt-induced axon regeneration. Deletion or inactivation of GSK3 $\beta$ promotes axon regeneration independently of the mTORC1 pathway, whereas constitutive activation of GSK3 $\beta$ reduces Akt-induced axon regeneration. $\mathrm{eIF}_{2} \mathrm{~B}_{\varepsilon}$ has been identified as a novel downstream effector of GSK3 $\beta$ and inactivation of eIF $2 B_{\varepsilon}$ reduces both GSK3 $\beta$ and Aktmediated effects on axon regeneration. Constitutive activation of $\mathrm{eIF} 2 \mathrm{~B}_{\varepsilon}$ is sufficient to promote axon regeneration, which 
reveals a key role of the Akt-GSK3 $\beta-\mathrm{eIF} 2 \mathrm{~B}_{\varepsilon}$ signaling module in regulating axon regeneration in the adult mammalian CNS (Guo et al., 2016). Akt activation is sufficient to promote optic nerve regeneration, but the regeneration is not as robust as that with PTEN deletion (Yang et al., 2014). Akt plays similar roles in the regeneration of lamprey CNS axons after SCI. In the present study, ChABC treatment greatly promoted axonal regeneration at 10 weeks after SCI. This was accompanied by widespread enhancement of Akt activation (pAkt-308) in the brain. IHC in individual identified RS neurons confirmed the increase in Akt phosphorylation. Taken together, these findings support the idea that enhanced activation of Akt is involved in the axon regeneration induced by $\mathrm{ChABC}$ treatment after SCI in lamprey.

On the other hand, Akt is also a critical pro-survival molecule in stressed cells. Previous studies showed that Akt can be phosphorylated by phosphoinositide 3-kinase (PI3K), and thereby protect tumor cells from death (Mayer and Arteaga, 2016). PI3K/Akt activation signals damage in neural tissues after SCI (Du et al., 2015; Wang et al., 2017; Qi et al., 2019). Rapamycin treatment can activate Akt via phosphorylation (O'Reilly et al., 2006; Wullschleger et al., 2006), which has been reported to suppress apoptosis in several models of ischemia reperfusion injury (Lee et al., 2004; Carloni et al., 2009). Interestingly, we found that $\mathrm{ChABC}$ treatment protects identified RS neurons from undergoing apoptosis at 2 and 11 weeks post-TX. The activation of Akt after ChABC treatment is consistent with the observed effect on neuronal survival. We conclude that Akt activation contributes to the beneficial effect of ChABC treatment in protecting the RS neurons from undergoing retrograde apoptosis after SCI. A report on axon regeneration after small peptideinduced inhibition of PTP $\sigma$ indicated that extracellular regulated kinases (Erks) also are involved in regeneration of peripheral axons (Yao et al., 2019). Another group of researchers specifically activated the ERK and Akt signaling pathways and performed a comprehensive study of neural regeneration in both PNS and CNS neurons in live Drosophila (Wang et al., 2020). They found that both ERK and Akt activations enhanced axon regeneration in the sensory neurons in Drosophila larvae (Wang et al., 2020). These reports strongly suggest that Erk might be involved in the downstream signaling pathway after ChABC treatment. We have also examined the roles of Erk in lamprey SCI model

\section{REFERENCES}

Barreiro-Iglesias, A., and Shifman, M. I. (2012). Use of fluorochrome-labeled inhibitors of caspases to detect neuronal apoptosis in the whole-mounted lamprey brain after spinal cord injury. Enzym. Res. 2012:835731. doi: 10.1155/ 2012/835731

Bradbury, E. J., Moon, L. D., Popat, R. J., King, V. R., Bennett, G. S., Patel, P. N., et al. (2002). Chondroitinase ABC promotes functional recovery after spinal cord injury. Nature 416, 636-640. doi: 10.1038/416636a

Bruckner, G., Grosche, J., Schmidt, S., Hartig, W., Margolis, R. U., Delpech, B., et al. (2000). Postnatal development of perineuronal nets in wild-type mice and in a mutant deficient in tenascin-R. J. Comp. Neurol. 428, 616-629. doi: 10.1002/1096-9861(20001225)428:4<616::aid-cne3<3.0.co;2-k

Busch, D. J., and Morgan, J. R. (2012). Synuclein accumulation is associated with cell-specific neuronal death after spinal cord injury. J. Comp. Neurol. 520, 1751-1771. doi: 10.1002/cne.23011 and explored the expression pattern after SCI in retrograde signaling (Jin et al., 2020). The specific role played by Erk after ChABC treatment in lamprey CNS is still unclear and under investigation.

\section{DATA AVAILABILITY STATEMENT}

The original contributions presented in the study are included in the article/supplementary material, further inquiries can be directed to the corresponding author/s.

\section{ETHICS STATEMENT}

The animal study was reviewed and approved by the Temple University Institutional Animal Care and Use Committee.

\section{AUTHOR CONTRIBUTIONS}

JH did the immunohistochemistry and FLICA staining. GZ prepared the in situ hybridization probes. WR and JH performed the in situ hybridization and acquired the images. L-QJ and $\mathrm{JH}$ and carried out the western blotting. JH and MS designed the experiments, carried out the data analysis, and drafted and prepared the manuscript. SL contributed to the experimental design and suggested specific experiments. All authors had full access to all the data in the study and take full responsibility for the integrity of the data and accuracy of the data analysis.

\section{FUNDING}

This work was supported by NIH grants R01NS092876, R01NS097846, and Shriners Research Foundation grants SHC85400, SHC-85220, SHC-85101 to MS; Shriners Research Foundation grant SHC-84293 to JH; NIH grants R01NS105961, 1R01NS079432, 1R01EY024575, and Shriners Research Foundation grants SHC-85100, SHC-86200-PHI-16, 85112-PHI18 to SL.

Busch, S. A., Horn, K. P., Silver, D. J., and Silver, J. (2009). Overcoming macrophage-mediated axonal dieback following CNS injury. J. Neurosci. 29, 9967-9976. doi: 10.1523/jneurosci.1151-09.200 9342

Cafferty, W. B., Bradbury, E. J., Lidierth, M., Jones, M., Duffy, P. J., Pezet, S., et al. (2008). Chondroitinase ABC-mediated plasticity of spinal sensory function. J. Neurosci. 11998-12009. doi: 10.1523/jneurosci.3877-08. 2008

Cafferty, W. B., Yang, S. H., Duffy, P. J., Li, S., and Strittmatter, S. M. (2007). Functional axonal regeneration through astrocytic scar genetically modified to digest chondroitin sulfate proteoglycans. J. Neurosci. 27, 2176-2185. doi: 10.1523/jneurosci.5176-06.2007

Carloni, S., Girelli, S., Buonocore, G., Longini, M., and Balduini, W. (2009). Simvastatin acutely reduces ischemic brain damage in the immature rat via Akt and CREB activation. Exp. Neurol. 220, 82-89. doi: 10.1016/j.expneurol.2009. 07.026 
D’Amelio, M., Cavallucci, V., and Cecconi, F. (2010). Neuronal caspase-3 signaling: not only cell death. Cell Death Differ. 17, 1104-1114. doi: 10.1038/cdd.2009.180

Davis, G. R. Jr., and McClellan, A. D. (1994). Long distance axonal regeneration of identified lamprey reticulospinal neurons. Exp. Neurol. 127, 94-105. doi: 10.1006/exnr.1994.1083

Deepa, S. S., Carulli, D., Galtrey, C., Rhodes, K., Fukuda, J., Mikami, T., et al. (2006). Composition of perineuronal net extracellular matrix in rat brain: a different disaccharide composition for the net-associated proteoglycans. J. Biol. Chem. 281, 17789-17800. doi: 10.1074/jbc.M600544200

Deliagina, T. G., Zelenin, P. V., Fagerstedt, P., Grillner, S., and Orlovsky, G. N. (2000). Activity of reticulospinal neurons during locomotion in the freely behaving lamprey. J. Neurophysiol. 83, 853-863.

Du, K., Zheng, S., Zhang, Q., Li, S., Gao, X., Wang, J., et al. (2015). Pten deletion promotes regrowth of corticospinal tract axons 1 year after spinal cord injury. J. Neurosci. 35, 9754-9763. doi: 10.1523/jneurosci.3637-14.2015

Fisher, D., Xing, B., Dill, J., Li, H., Hoang, H. H., Zhao, Z., et al. (2011). Leukocyte common antigen-related phosphatase is a functional receptor for chondroitin sulfate proteoglycan axon growth inhibitors. J. Neurosci. 31, 14051-14066. doi: 10.1523/jneurosci.1737-11.2011

Friedlander, D. R., Milev, P., Karthikeyan, L., Margolis, R. K., Margolis, R. U., and Grumet, M. (1994). The neuronal chondroitin sulfate proteoglycan neurocan binds to the neural cell adhesion molecules Ng-CAM/L1/NILE and N-CAM, and inhibits neuronal adhesion and neurite outgrowth. J. Cell Biol. 125, 669680. doi: $10.1083 /$ jcb.125.3.669

Galtrey, C. M., Asher, R. A., Nothias, F., and Fawcett, J. W. (2007). Promoting plasticity in the spinal cord with chondroitinase improves functional recovery after peripheral nerve repair. Brain 130(Pt. 4), 926-939. doi: 10.1093/brain/ awl372

Guo, X., Snider, W. D., and Chen, B. (2016). GSK3beta regulates AKT-induced central nervous system axon regeneration via an eIF2Bepsilon-dependent, mTORC1-independent pathway. Elife 5:e11903. doi: 10.7554/eLife.11903

Hower, A. E., Beltran, P. J., and Bixby, J. L. (2009). Dimerization of tyrosine phosphatase PTPRO decreases its activity and ability to inactivate TrkC. J. Neurochem. 110, 1635-1647. doi: 10.1111/j.1471-4159.2009.06261.x

Hu, J., Zhang, G., and Selzer, M. E. (2013). Activated caspase detection in living tissue combined with subsequent retrograde labeling, immunohistochemistry or in situ hybridization in whole-mounted lamprey brains. J. Neurosci. Methods 220, 92-98. doi: 10.1016/j.jneumeth.2013.08.016

Hu, J., Zhang, G., Rodemer, W., Jin, L. Q., Shifman, M., and Selzer, M. E. (2017). The role of RhoA in retrograde neuronal death and axon regeneration after spinal cord injury. Neurobiol. Dis. 98, 25-35. doi: 10.1016/j.nbd.2016.11.006

Jacobs, A. J., Swain, G. P., Snedeker, J. A., Pijak, D. S., Gladstone, L. J., and Selzer, M. E. (1997). Recovery of neurofilament expression selectively in regenerating reticulospinal neurons. J. Neurosci. 17, 5206-5220.

Jin, L. Q., John, B. H., Hu, J., and Selzer, M. E. (2020). Activated erk is an early retrograde signal after spinal cord injury in the lamprey. Front. Neurosci. 14:580692. doi: $10.3389 /$ fnins.2020.580692

Jin, L. Q., Zhang, G., Jamison, C. Jr., Takano, H., Haydon, P. G., and Selzer, M. E. (2009). Axon regeneration in the absence of growth cones: acceleration by cyclic AMP. J. Comp. Neurol. 515, 295-312. doi: 10.1002/cne.22057

Kuo, C. T., Zhu, S., Younger, S., Jan, L. Y., and Jan, Y. N. (2006). Identification of E2/E3 ubiquitinating enzymes and caspase activity regulating Drosophila sensory neuron dendrite pruning. Neuron 51, 283-290. doi: 10.1016/j.neuron. 2006.07.014

Lang, B. T., Cregg, J. M., DePaul, M. A., Tran, A. P., Xu, K., Dyck, S. M., et al. (2015). Modulation of the proteoglycan receptor PTPsigma promotes recovery after spinal cord injury. Nature 518, 404-408. doi: 10.1038/nature13974

Lee, H. T., Chang, Y. C., Wang, L. Y., Wang, S. T., Huang, C. C., and Ho, C. J. (2004). cAMP response element-binding protein activation in ligation preconditioning in neonatal brain. Ann. Neurol. 56, 611-623. doi: 10.1002/ana. 20259

Li, Z., Jo, J., Jia, J. M., Lo, S. C., Whitcomb, D. J., Jiao, S., et al. (2010). Caspase-3 activation via mitochondria is required for long-term depression and AMPA receptor internalization. Cell 141, 859-871. doi: 10.1016/j.cell.2010. 03.053

Liu, K., Lu, Y., Lee, J. K., Samara, R., Willenberg, R., Sears-Kraxberger, I., et al. (2010). PTEN deletion enhances the regenerative ability of adult corticospinal neurons. Nat. Neurosci. 13, 1075-1081. doi: 10.1038/nn.2603
Liu, K., Tedeschi, A., Park, K. K., and He, Z. (2011). Neuronal intrinsic mechanisms of axon regeneration. Annu. Rev. Neurosci. 34, 131-152. doi: 10.1146/annurevneuro-061010-113723

Lu, C., Wang, Y., Furukawa, K., Fu, W., Ouyang, X., and Mattson, M. P. (2006). Evidence that caspase-1 is a negative regulator of AMPA receptor-mediated long-term potentiation at hippocampal synapses. J. Neurochem. 97, 1104-1110. doi: 10.1111/j.1471-4159.2006.03800.x

Mayer, I. A., and Arteaga, C. L. (2016). The PI3K/AKT pathway as a target for cancer treatment. Annu. Rev. Med. 67, 11-28. doi: 10.1146/annurev-med062913-051343

McKeon, R. J., Schreiber, R. C., Rudge, J. S., and Silver, J. (1991). Reduction of neurite outgrowth in a model of glial scarring following CNS injury is correlated with the expression of inhibitory molecules on reactive astrocytes. J. Neurosci. 11, 3398-3411. doi: 10.1523/JNEUROSCI.11-11-03398.1991

Monnier, P. P., Sierra, A., Schwab, J. M., Henke-Fahle, S., and Mueller, B. K. (2003). The Rho/ROCK pathway mediates neurite growth-inhibitory activity associated with the chondroitin sulfate proteoglycans of the CNS glial scar. Mol. Cell. Neurosci. 22, 319-330.

Murakami, Y., Pasqualetti, M., Takio, Y., Hirano, S., Rijli, F. M., and Kuratani, S. (2004). development of reticulospinal and branchiomotor neurons in lamprey: insights into the evolution of the vertebrate hindbrain. Development 131, 983-995. doi: 10.1242/dev.00986

O'Reilly, K. E., Rojo, F., She, Q. B., Solit, D., Mills, G. B., Smith, D., et al. (2006). mTOR inhibition induces upstream receptor tyrosine kinase signaling and activates Akt. Cancer Res. 66, 1500-1508. doi: 10.1158/0008-5472.can-05-2925

Park, K. K., Liu, K., Hu, Y., Smith, P. D., Wang, C., Cai, B., et al. (2008). Promoting axon regeneration in the adult CNS by modulation of the PTEN/mTOR pathway. Science 322, 963-966. doi: 10.1126/science.1161566

Qi, L., Jiang-Hua, M., Ge-Liang, H., Qing, C., and Ya-Ming, L. (2019). MiR-34a inhibits spinal cord injury and blocks spinal cord neuron apoptosis by activating phatidylinositol 3-kinase (PI3K)/AKT pathway through targeting CD47. Curr. Neurovasc. Res. 16, 373-381. doi: 10.2174/1567202616666190906102343

Rodemer, W., Zhang, G., Sinitsa, I., Hu, J., Jin, L. Q., Li, S., et al. (2020). PTP $\sigma$ knockdown in lampreys impairs reticulospinal axon regeneration and neuronal survival after spinal cord injury. Front. Cell Neurosci. 14:61. doi: 10.3389/fncel. 2020.00061

Sharma, K., Selzer, M. E., and Li, S. (2012). Scar-mediated inhibition and CSPG receptors in the CNS. Exp. Neurol. 237, 370-378. doi: 10.1016/j.expneurol.2012. 07.009

Shen, Y., Tenney, A. P., Busch, S. A., Horn, K. P., Cuascut, F. X., Liu, K., et al. (2009). PTPsigma is a receptor for chondroitin sulfate proteoglycan, an inhibitor of neural regeneration. Science 326, 592-596. doi: 10.1126/science. 1178310

Shifman, M. I., Zhang, G., and Selzer, M. E. (2008). Delayed death of identified reticulospinal neurons after spinal cord injury in lampreys. J. Comp. Neurol. 510, 269-282. doi: 10.1002/cne.21789

Snow, D. M., Lemmon, V., Carrino, D. A., Caplan, A. I., and Silver, J. (1990). Sulfated proteoglycans in astroglial barriers inhibit neurite outgrowth in vitro. Exp. Neurol. 109, 111-130. doi: 10.1016/s0014-4886(05)80013-5

Starkey, M. L., Bartus, K., Barritt, A. W., and Bradbury, E. J. (2012). Chondroitinase $\mathrm{ABC}$ promotes compensatory sprouting of the intact corticospinal tract and recovery of forelimb function following unilateral pyramidotomy in adult mice. Eur. J. Neurosci. 36, 3665-3678. doi: 10.1111/ejn.12017

Swain, G. P., Jacobs, A. J., Frei, E., and Selzer, M. E. (1994). A method for in situ hybridization in wholemounted lamprey brain: neurofilament expression in larvae and adults. Exp. Neurol. 126, 256-269. doi: 10.1006/exnr.1994. 1063

Wang, Q., Fan, H., Li, F., Skeeters, S. S., Krishnamurthy, V. V., Song, Y., et al. (2020). Optical control of ERK and AKT signaling promotes axon regeneration and functional recovery of PNS and CNS in Drosophila. Elife 9:e57395. doi: 10.7554/eLife. 57395

Wang, Z., Zhou, L., Zheng, X., Chen, G., Pan, R., Li, J., et al. (2017). Autophagy protects against PI3K/Akt/mTOR-mediated apoptosis of spinal cord neurons after mechanical injury. Neurosci. Lett. 656, 158-164. doi: 10.1016/j.neulet.2017. 07.036

Williams, D. W., Kondo, S., Krzyzanowska, A., Hiromi, Y., and Truman, J. W. (2006). Local caspase activity directs engulfment of dendrites during pruning. Nat. Neurosci. 9, 1234-1236. doi: 10.1038/nn1774 
Wullschleger, S., Loewith, R., and Hall, M. N. (2006). TOR signaling in growth and metabolism. Cell 124, 471-484. doi: 10.1016/j.cell.2006.01.016

Xu, B., Park, D., Ohtake, Y., Li, H., Hayat, U., Liu, J., et al. (2015). Role of CSPG receptor LAR phosphatase in restricting axon regeneration after CNS injury. Neurobiol. Dis. 73, 36-48. doi: 10.1016/j.nbd.2014.08.030

Yang, L., Miao, L., Liang, F., Huang, H., Teng, X., Li, S., et al. (2014). The mTORC1 effectors $\mathrm{S} 6 \mathrm{~K} 1$ and $4 \mathrm{E}-\mathrm{BP}$ play different roles in CNS axon regeneration. Nat. Commun. 5:5416. doi: 10.1038/ncomms6416

Yao, M., Sun, H., Yuan, Q., Li, N., Li, H., Tang, Y., et al. (2019). Targeting proteoglycan receptor PTP $\sigma$ restores sensory function after spinal cord dorsal root injury by activation of Erks/CREB signaling pathway. Neuropharmacology 144, 208-218. doi: 10.1016/j.neuropharm.2018. 10.035

Zhang, G., Hu, J., Li, S., Huang, L., and Selzer, M. E. (2014). Selective expression of CSPG receptors PTPsigma and LAR in poorly regenerating reticulospinal neurons of lamprey. J. Comp. Neurol. 522, 2209-2229. doi: 10.1002/cne.23529
Zhong, J., Lan, C., Zhang, C., Yang, Y., Chen, W. X., Zhang, K. Y., et al. (2019). Chondroitin sulfate proteoglycan represses neural stem/progenitor cells migration via PTPsigma/alpha-actinin4 signaling pathway. J. Cell. Biochem. 120, 11008-11021. doi: $10.1002 /$ jcb. 28379

Conflict of Interest: The authors declare that the research was conducted in the absence of any commercial or financial relationships that could be construed as a potential conflict of interest.

Copyright (c) $2021 \mathrm{Hu}$, Rodemer, Zhang, Jin, Li and Selzer. This is an open-access article distributed under the terms of the Creative Commons Attribution License (CC BY). The use, distribution or reproduction in other forums is permitted, provided the original author(s) and the copyright owner(s) are credited and that the original publication in this journal is cited, in accordance with accepted academic practice. No use, distribution or reproduction is permitted which does not comply with these terms. 